This item was submitted to Loughborough's Research Repository by the author.

Items in Figshare are protected by copyright, with all rights reserved, unless otherwise indicated.

\title{
Simulations of the dispersion of reactive pollutants in a street canyon, considering different chemical mechanisms and micromixing
}

PLEASE CITE THE PUBLISHED VERSION

http://dx.doi.org/10.1016/j.atmosenv.2008.07.033

PUBLISHER

(C) Elsevier Ltd.

VERSION

AM (Accepted Manuscript)

LICENCE

CC BY-NC-ND 4.0

\section{REPOSITORY RECORD}

Garmory, Andrew, I.S. Kim, R.E. Britter, and E. Mastorakos. 2012. "Simulations of the Dispersion of Reactive Pollutants in a Street Canyon, Considering Different Chemical Mechanisms and Micromixing”. figshare. https://hdl.handle.net/2134/11118. 
This item was submitted to Loughborough's Institutional Repository (https://dspace.lboro.ac.uk/) by the author and is made available under the following Creative Commons Licence conditions.

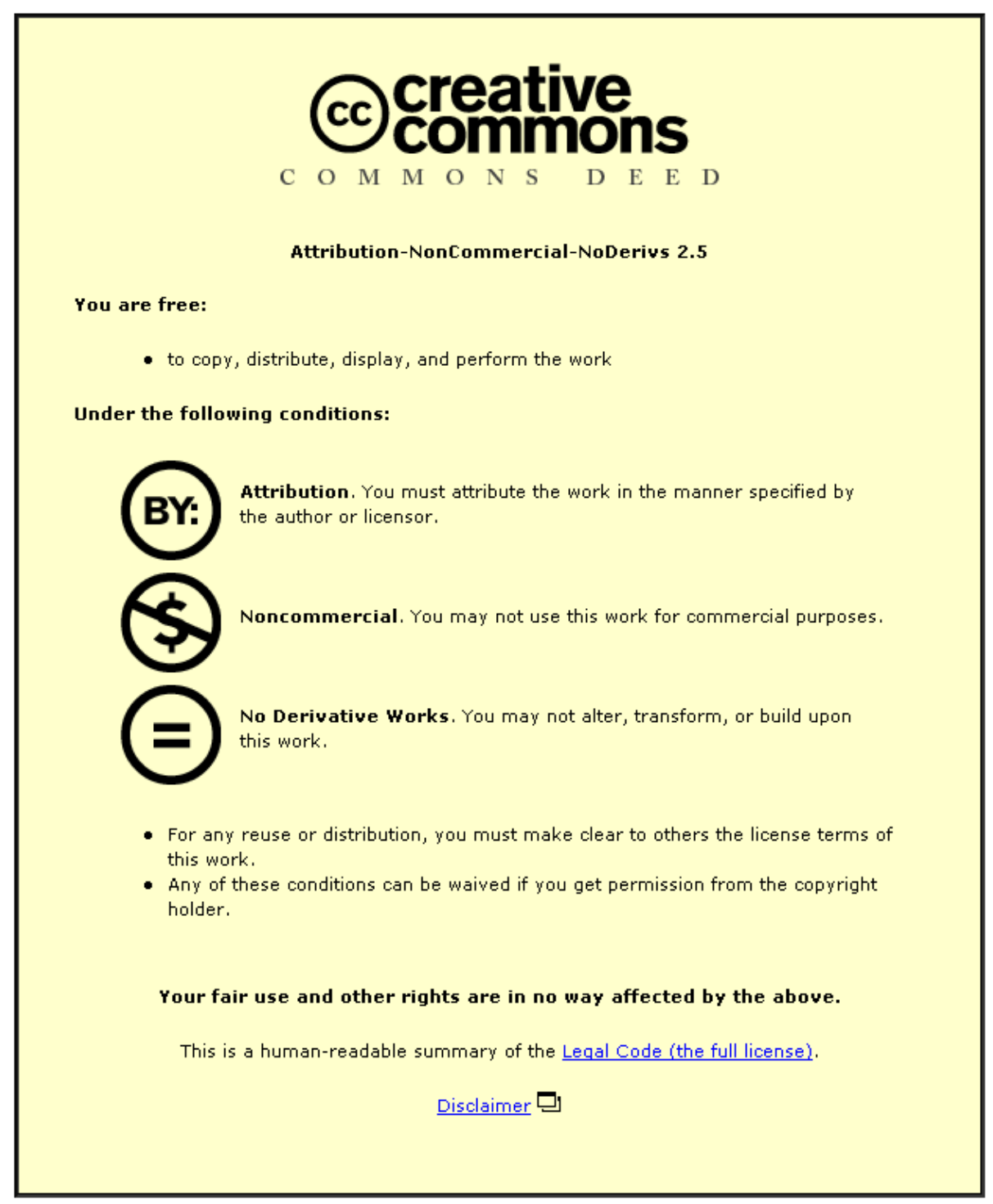

For the full text of this licence, please go to: http://creativecommons.org/licenses/by-nc-nd/2.5/ 


\title{
Simulations of The Dispersion of Reactive \\ Pollutants In A Street Canyon, Considering \\ Different Chemical Mechanisms and
}

Micromixing

\begin{abstract}
A. Garmory ${ }^{\text {a,* }}$, I. S. Kim ${ }^{\text {b }}$, R. E. Britter ${ }^{a}$ and E. Mastorakos ${ }^{\text {a }}$
${ }^{a}$ Hopkinson Laboratory, Department of Engineering, University of Cambridge, UK

${ }^{\mathrm{b}}$ Current Address, Siemens Industrial Turbomachinery, Lincoln, UK
\end{abstract}

\begin{abstract}
The Stochastic Fields (SF) or Field Monte Carlo method has been used to model the dispersion of reactive scalars in a street canyon, using a simple chemistry and the CBM-IV mechanism. SF is a Probability Density Function (PDF) method which allows both means and variances of the scalars to be calculated as well as considering the effect of segregation on reaction rates. It was found that the variance of reactive scalars such as $\mathrm{NO}_{2}$ was very high in the mixing region at roof top level with rms values of the order of the mean values. The effect of segregation on major species such as $\mathrm{O}_{3}$ was found to be very small using either mechanism, however some radical species in CBM-IV showed a significant difference. These were found to be the seven species with the fastest chemical timescales. The calculated photostationary state defect was also found to be in error when segregation is neglected.
\end{abstract}

Key words: Turbulent reacting flows; Stochastic Fields; Probability Density Function; Pollution; Urban. 


\section{Introduction}

The street canyon has for many years been an active area of study in air quality modelling, both in terms of predicting actual roadside exposure to pollution and as a more theoretical test-case for using different numerical models. Experimental data has been obtained both from field studies (Rotach, 1995; Costabile and Allegrini, 2007) and from wind-tunnel (Pavageau and Schatzmann, 1999) and water tank models (Caton et al., 2003). Computational modelling has been carried out in order to investigate the fluid mechanics and the dispersion of pollutants in and around the canyon (Chan et al., 2001; Baik and Kim, 2002; Jeong and Andrews, 2002). Work has also been carried out which attempts to quantify the exchange of mass and momentum at rooftop level using an exchange velocity in order to provide a model that can be applied to a real-world situations (Hamlyn and Britter, 2005). Walton and Cheng (2002) and Lui and Barth (2002) have used a LES calculation to predict the dispersion and fluctuation of an inert pollutant in a street canyon. Dixon and Tomlin (2007) have used a Lagrangian stochastic model for the same purpose.

Recent studies have focused on the turbulent dispersion of reactive pollutants in the street canyon setting. Baker et al. (2004) have carried out a LES study of a street canyon using a one-step, reversible $\mathrm{NO}, \mathrm{NO}_{2}$ and $\mathrm{O}_{3}$ chemistry using a constant temperature in the canyon. They found that spatial variation of species within the canyon was significant. Baik et al. (2007), on the other hand, have used RANS modelling to predict the flow field in the canyon while using a similar chemistry to Baker et al. (2004). They performed a budget analysis of the terms in the advection-diffusion-reaction equation, which showed that

\footnotetext{
* Corresponding author.

Email address: ag310@cam.ac.uk (A. Garmory).
} 
the chemical reaction term is comparable to the advection and diffusion terms for $\mathrm{O}_{3}$. Both these studies found that the air composition is close to chemical equilibrium within the canyon but is far from equilibrium where polluted air from the canyon is mixed with clean air at the top of the canyon.

Most reactive pollutant dispersion models so far have not included the effect of turbulence on reaction rates. Due to the non-linearity of the Arrhenius term and the effect of non-zero covariances between reactants evaluating mean reaction rates as a function only of mean concentrations and temperature may not yield a correct value. The fluctuations from the mean (either spatially or temporally) may need to be considered. The presence of fluctuations from the mean is known as segregation, while their decay due to molecular diffusion at the smallest scales of turbulence is often called micromixing. Whether the segregation has a significant effect on the reaction rate will depend on how rapidly micromixing destroys the segregation compared to the reaction speed. This is characterised by the Damköhler number, $D a$, defined as the ratio of mixing timescale to chemical timescale $D a=T_{\text {phys }} / T_{\text {chem }}$. For further discussion of this see Garmory et al. (2006). The above comments apply equally to RANS and LES calculations, the difference being that in the latter case the sub-grid segregation is expected to be smaller, but still not negligible in principle.

In this work we use the Field Monte Carlo method to simulate the dispersion of reactive pollutants within a street canyon and also to assess what effect segregation and micromixing have on the reactions. This is done using the simple $\mathrm{NO}, \mathrm{NO}_{2}$ and $\mathrm{O}_{3}$ chemistry used in Baik et al. (2007) and also using the CBM-IV mechanism, which is a more complex chemistry comprising 28 species (Gery et al., 1989). The Field Monte Carlo method, also called the Stochastic Fields method, is a transported PDF method developed independently by 
Valiño (1998) and Sabel'nikov and Soulard (2005b). Rather than using the motion of notional particles through the flow this method solves stochastic partial differential equations (spde), derived from the modelled transported PDF equation (Fox, 2003), for a number of scalar fields extending across the spatial domain. A spde is solved for each scalar in each field, if the values for a particular scalar are taken at a point in space across all fields then the ensemble is statistically equivalent to the flow at that point. By doing this the effect of fluctuations on the reaction rates are calculated directly with no need for closure models for the mean reaction rate, which constitutes the greatest difficulty in turbulent reacting flows. The advantage of the field based method is that it is easily coupled with existing CFD techniques and is hence straightforward to implement in practical scenarios. In this work, the reactions have no effect on the density due to their negligible heat release, which allows us to pre-calculate the flow and then use a 'frozen' flow field for the subsequent reactive scalar calculations.

\section{Formulation}

\subsection{The Stochastic Fields Method}

The Stochastic Fields, or Field Monte Carlo, method is a transported joint composition PDF method developed for the simulation of turbulent reacting flows (Valiño, 1998; Sabel'nikov and Soulard, 2005b). A number of 'fields' extending across the whole spatial domain of the simulation are used which contain values for each scalar at every node on an Eulerian grid. The evolution of each field takes place according to a governing spde derived from the scalar PDF transport equation. The Ito SPDE as derived by Valiño (1998), using 
the Interaction by Exchange with the Mean (IEM) closure for micromixing, is:

$$
\begin{aligned}
d \tau_{i}^{f} & =-U_{k} \frac{\partial \tau_{i}^{f}}{\partial x_{k}} d t+\frac{\partial}{\partial x_{k}}\left(K \frac{\partial \tau_{i}^{f}}{\partial x_{k}}\right) d t \\
& +\dot{w}\left(\tau_{1}^{f}, \tau_{2}^{f}, \ldots, \tau_{I}^{f}\right) d t+(2 K)^{1 / 2} \frac{\partial \tau_{i}^{f}}{\partial x_{k}} d W_{k}^{f}-\frac{\tau_{i}^{f}-\bar{\phi}_{i}}{T_{\text {eddy }}} d t
\end{aligned}
$$

where $\tau_{i}^{f}$ is the value of scalar $i$ in field $f(f=1, \ldots, F)$ and $\bar{\phi}_{i}$ is the local mean of scalar $i$. $U_{k}$ is the mean velocity, $K$ is the combined molecular and turbulent diffusivity, $T_{e d d y}$ is a characteristic timescale of turbulent scalar mixing used in the IEM model. As this is an Ito $\operatorname{SPDE}(2 K)^{1 / 2} \frac{\partial \tau_{i}^{f}}{\partial x_{k}}$ must be evaluted only once per timestep to ensure that it is independent of $d W_{k}^{f}$ (Gardiner, 2004). The PDF is then represented by the ensemble of $F$ stochastic fields $\tau^{f}$ which contain values for each scalar at each point throughout the flow. As this is a joint scalar PDF method and not a joint velocity-scalar PDF method (Pope, 1994) the velocity, turbulence and timescale must be provided from an external source, which in this case is a CFD solution. The SPDE solved here, Eq. (1), is in non-divergent form, Soulard and Sabel'nikov (2006) derive an SPDE in divergent form which gives results statistically equivalent to those used here. While not employed here it is thought that this divergent form may offer advantages in implementation using conservative numerical schemes. The Stochastic Fields method has to date been used to model combustion (Sabel'nikov and Soulard, 2005a; Mustata et al., 2006). We have previously used the method to simulate a $\mathrm{NO}_{x} / \mathrm{O}_{3}$ reacting plume in laboratory conditions (Garmory et al., 2006) and found excellent agreement with experimental data for mean and rms values. The method was also extended to the simulation of a jet engine exhaust plume in Garmory et al. (2008). The reader is referred to these papers for further discussion of the method. 


\subsection{Numerical Methods}

In this paper we use the same procedure for solving the Stochastic Fields equation as in our earlier paper Garmory et al. (2008). As a separate CFD calculation of the flow is required, we coupled our Field Monte Carlo method with a commercial CFD package, FLUENT. This was used not only to calculate the flow field, but also, by using an operator-splitting method, the spatial transport terms in Eq. (1) for each time-step. The advantages of coupling with a commercial CFD package are that there is no difficulty in exporting velocity field data to the reacting flow code and also that all the grid making and post-processing tools already available in FLUENT (or any other CFD code) can be used with this problem.

An operator-splitting procedure is used where in the first fractional step FLUENT's User Defined Scalars (UDS) option is used to solve for the transport terms in Eq. (1) for each scalar in each field using an iterative implicit method. The second step is to perform the random, or Wiener, step for each scalar in each field using $\Delta W_{k}^{f}=\xi_{k}^{f} \Delta t^{1 / 2}$ where $\xi_{k}^{f}$ is a Gaussian random number with zero mean and unity variance (Gardiner, 2004). An independent value of $\xi$ is required for each spatial component in each field. A single-step, explicit method has been used to to integrate this step so that the integrand of the random term remains independent of $\Delta W$ in order to correctly calculate the Ito integral (Garmory, 2007). At this stage $\bar{\phi}_{i}$ is calculated for each scalar at each grid node by taking the mean of the values in all fields at that node, this is then used in the micromixing term which is integrated along with the chemistry by a stiff ODE solver which enables the use of chemistries with a wide range of timescales. This operator-splitting procedure used to couple the SF code with the CFD is the same as that used in Garmory et al. (2008) in 
all respects except, firstly, that here transport is solved in 2D rather than 3D, and secondly, that the stiff ODE solver VODPK (Byrne, 1992) is used to solve for the chemistry step.

We assume a turbulent Schmidt number of unity and thereby set the turbulent diffusivity equal to the effective dynamic viscosity divided by the density, which are calculated by FLUENT in the CFD solution. The turbulent mixing timescale, $T_{\text {eddy }}$, was found by setting it equal to mechanical turbulence timescale $T_{\text {vel }}=k / \epsilon$. This is valid for homogeneous turbulence with no scalar gradient and while it is not necessarily valid away from these conditions it has been found that the ratio stays close to unity in many other situations (Cassiani et al., 2005). This assumption has previously been used to accurately predict the variance of a passive tracer in a laboratory plume (Garmory et al., 2006). In order to have full control over the timestep, the simulation is run as an unsteady case, so advection-diffusion is solved for all scalars for one timestep before a user defined subroutine is used to perform the remaining fractional steps and update the scalar values before the next timestep.

\subsection{Model Problem}

The velocity and turbulence fields were calculated using a 2D RANS method with the $k-\epsilon$ model in FLUENT. The parameters used in the solution are presented in Table 1. The two-dimensional CFD domain consisted of seven identical evenly spaced street canyons of width $20 \mathrm{~m}$ and height $24 \mathrm{~m}$. These are the same dimensions as used in Walton and Cheng (2002). The total height of the domain was $100 \mathrm{~m}$ and it extended 50m upstream of the first 'building.' This grid contained a total of 79,680 grid cells. An inlet boundary layer profile for velocity was used at the upstream boundary, which obeys a $(1 / 7)^{\text {th }}$ power- 
law:

$$
u(y)=\frac{8}{7} U_{b u l k}\left(\frac{y}{d}\right)^{1 / 7}
$$

The bulk velocity, $U_{\text {bulk}}$, was specified as $5 \mathrm{~m} \mathrm{~s}^{-1}$, and $d=50 \mathrm{~m}$. A turbulence intensity of $10 \%$ and a length scale of $100 \mathrm{~m}$ are used as the turbulence boundary conditions. The downstream boundary was set to be an outflow. The top of the domain was set as a symmetry boundary condition, but this was sufficiently far above the canyons that its effect is negligible.

Seven adjacent canyons were used in the CFD solution so that within the sixth canyon a typical, fully-developed single vortex is produced. The velocity field in this canyon should depend little on the details of the velocity and turbulence inlet profiles at the entrance to the CFD grid as the boundary layer is allowed to develop over five canyons prior to reaching the one of interest. The Stochastic Fields calculations were performed using a domain that includes only the sixth canyon and extends $20 \mathrm{~m}$ along the neighbouring rooftops. The height of this grid was $70 \mathrm{~m}$ in total and a grid resolution half of that for the CFD solution was used, giving a total of 14,700 cells in this grid. A schematic diagram of the canyon, including some salient features of the problem, is shown in Fig. 1. The velocity and turbulence fields were fixed as those produced in the larger CFD calculation by turning off the flow and turbulence solvers in FLUENT for the duration of the SF calculation. This is possible as the reactions involved do not produce sufficient heat to affect the density of the flow. The velocity field in the canyon is shown in Fig. 2. A background concentration for each species was set at the inlet (left hand edge, above the building level) and initially at all points in the grid. The emission source was defined by setting a fixed value for each species in a $0.6 \times 0.3 \mathrm{~m}$ region consisting of 4 grid cells centred on a point on the centre-line of the 
canyon $0.45 \mathrm{~m}$ above the ground. The time-step used was $0.1 \mathrm{~s}$ and it was found that the solution did not change appreciably after 100s. 60 fields were used with the simple Baik et al chemistry but only 14 for the CBM mechanism

due to constraints of user defined memory in FLUENT. Means, variances and other statistical data are then found at each grid node by averaging over the values in each field at a single point in time.

\section{Results and Discussion}

\subsection{Inert Mixing}

The inert dispersion of pollutants within the canyon can be characterised by the mean and variance of mixture fraction. This is simulated by using an extra scalar with no chemical source term in each field when using the simple chemistry with 60 fields and setting the source and background values to be one and zero respectively. The mean and rms calculated in this way are shown in Fig. 3, rapid mixing around the source and at the top of the canyon leads to high rms values there. The results here cannot be compared directly with the wind tunnel results of Pavageau and Schatzmann (1999), which provides mean and rms data for an inert scalar, as a slightly different canyon aspect ratio has been used. However the trends seen here follow those seen in their data which is as would be expected as PDF simulations using $k-\epsilon$ modelling for the flow field has been widely used and validated, including recently by Dixon and Tomlin (2007).

Further validation of the method used here is provided by Fig. 4 in which we follow Walton and Cheng (2002) by plotting mean mixture fraction concentration on the leeward and windward walls normalised by the value found 
at ground level. This is plotted along with data taken from Hoydysh and Dabberdt (1988) who collected wind tunnel data for an inert scalar in street canyons of several different configurations. As can be seen in Fig. 4 we find excellent agreement between our data and their measurements.

\subsection{Simple Chemistry - Statistics}

For the simple chemistry (Baik et al., 2007) we used a background $\mathrm{O}_{3}$ concentration of $30 \mathrm{ppb}, \mathrm{NO}$ and $\mathrm{NO}_{2}$ were set to $0.05 \mathrm{ppb}$ and $0.2 \mathrm{ppb}$ respectively. At the emission source the $O_{3}$ level was set to zero with constant values for $\mathrm{NO}$ of $1000 \mathrm{ppb}$ and $10 \mathrm{ppb}$ for $\mathrm{NO}_{2}$. With the velocity field and source size used here this corresponds to a $N O$ emission rate of approximately $200 \mu \mathrm{g}$ $\mathrm{m}^{-1} \mathrm{~s}^{-1}$. This is in the region of medium traffic (Baker et al., 2004). The $\mathrm{NO}_{2}$ level is low but it allows us to observe more clearly its production by chemical reaction in the canyon. Figs. 5(a) \& (b) show the mean and rms values for $\mathrm{NO}_{2}$ in and above the canyon as calculated by the SF method. The relatively long residence time allows the reaction of $\mathrm{NO}$ and $\mathrm{O}_{3}$ to build up the $\mathrm{NO}_{2}$ in the centre of the canyon to a level of approximately $23 \mathrm{ppb}$, which is more than double the level in our source. The mixing layer at the building height introduces relatively clean air into the canyon along the windward (right hand) wall, while polluted air is transported out of the domain in the downwind direction. As expected, the highest variance is observed where the scalar gradient is largest hence the variance is particularly large around the source and in the mixing layer.

In the mixing layer the rms is of the order of the mean concentration in this region. This high rms in the mixing layer agrees with the results seen in Dixon and Tomlin (2007). One of the advantages of using a PDF method is that the 
statistical distribution of the scalars can be investigated in greater detail. The skewness and kurtosis excess of the distribution are defined as:

$$
\begin{aligned}
\operatorname{skew}\left(\tau_{i}\right) & =\frac{\sqrt{F} \sum_{f=1}^{F}\left(\tau_{i}^{f}-\bar{\tau}_{i}\right)^{3}}{\left(\sum_{f=1}^{F}\left(\tau_{i}^{f}-\bar{\tau}_{i}\right)^{2}\right)^{3 / 2}} \\
\operatorname{kur}\left(\tau_{i}\right) & =\frac{F \sum_{f=1}^{F}\left(\tau_{i}^{f}-\overline{\tau_{i}}\right)^{4}}{\left(\sum_{f=1}^{F}\left(\tau_{i}^{f}-\overline{\tau_{i}}\right)^{2}\right)^{2}}-3
\end{aligned}
$$

where again $\tau_{i}^{f}$ is the value of species $i$ in field $f$ of a total of $F$ fields. Figs. 5(c) $\&$ (d) show contours of the skewness and kurtosis excess of $\mathrm{NO}_{2}$ in and above the canyon. It can be seen that there is a region of positive skewness above the mixing layer, which indicates that the $\mathrm{NO}_{2}$ concentration will usually be low with occasionally much higher concentration. There is a corresponding region of negative skewness just below the mixing layer which extends round the canyon vortex, surrounding the region of high $\mathrm{NO}_{2}$. In these areas the concentration will generally be high with occasional lower values.

Fig. 5(d) shows contours of kurtosis excess with only negative parts shown to increase clarity. A strongly negative region is observed at the level of the roof-tops indicating that the PDF here is not clustered around the mean. For reference, a two delta-function distribution would have a kurtosis excess of -2. These results are consistent with the idea that the chemical composition found in the mixing layer will, at a given instant, be likely to retain much of the composition of either 'in canyon' or 'out of canyon' air rather than a mixture of the two. This is illustrated by Fig. 6 which shows the PDF of $\mathrm{NO}_{2}$, as calculated from three SF simulations, at a point at roof-top level in the centre of the canyon. With only 60 samples in total the number of samples in each bin is relatively small and hence there will be a relatively large amount of statistical noise. Because of this the PDF's as calculated from 
three simulations using different random number sequences are used. It can be seen that while there is some noise the overall shape for the PDF here can be observed.

Fig. 7 shows mean and rms concentrations for $\mathrm{O}_{3}$ along a vertical line extending through the centre of the canyon. These results were produced using the SF method, Eq. (1), and also, by using a single field and dropping the random and micromixing terms, i.e. a plain advection-diffusion-reaction method. Once again the high variance at roof-top level is seen. It can be seen that the two solutions for $\mathrm{O}_{3}$ do not differ by more than the statistical noise seen in the inert scalar results. Similar results are seen for $\mathrm{NO}$ and $\mathrm{NO}_{2}$, indicating that segregation does not play a significant role in this situation, for these species.

This conclusion is supported by consideration of the Damköhler number. This is the ratio of physical mixing timescale to chemical timescale. A physical timescale has already been calculated in the CFD solution and is assumed to be the same for each species. An approximate method of obtaining a chemical timescale for each species is to find the rate at which it relaxes back to a local equilibrium value (Neophytou et al., 2004; Garmory et al., 2008). This rate can easily be extracted from the VODPK solver at each cell to give the distribution of timescale and hence $D a=T_{\text {eddy }} / T_{\text {chem }}$ can be calculated across the domain for each species. This is done for each field and the mean taken. It is found that the highest $\mathrm{Da}$ is for $\mathrm{O}_{3}$, which takes a maximum value of approximately three. This maximum value occurs in the centre of the canyon where the gradients are small and hence variance is low. In previous work, where timescales and Da were defined in the same way as here, (Garmory et al., 2008) segregation effects were seen when $D a \geq \sim 5$ in regions where scalar gradients were found. Therefore segregation effects should not be expected in this situation. 


\subsection{Simple Chemistry - Photostationary State Defect}

The photostationary state defect, $\delta_{p h}$, is defined in Baik et al. (2007) as:

$$
\delta_{p h}=\left(\frac{k_{1} X_{N O} X_{O_{3}}}{J_{N O_{2}} X_{N O_{2}}}-1\right) \times 100
$$

where $k_{1}$ is the reaction rate of the forward $\mathrm{NO}+\mathrm{O}_{3} \rightarrow \mathrm{NO}_{2}+\mathrm{O}_{2}$ reaction and $J_{\mathrm{NO}_{2}}$ is the photolysis rate of $\mathrm{NO}_{2} \rightarrow \mathrm{NO}+\mathrm{O}$. As the subsequent combination of the oxygen radical with an oxygen molecule to form ozone happens very quickly, a dynamic equilibrium, or photostationary state, will be achieved between $\mathrm{NO}, \mathrm{NO}_{2}$ and $\mathrm{O}_{3}$ if they are left unperturbed. When this occurs the above expression takes a value of zero. A departure from zero indicates how far the $\mathrm{NO}_{x} / \mathrm{O}_{3}$ system has moved from equilibrium with positive values indicating an excess of $\mathrm{NO}$ and $\mathrm{O}_{3}$. The idea of the photostationary state defect is also used in air quality measurement as a means of detecting other pollutants (Carpenter et al., 1998); departures from the photostationary state value in unperturbed air will reveal the presence of other pollutants. However in our simple chemistry modelling case, where there are no other species used, values of $\delta_{p h}$ away from zero indicate only that the reversible system is not in steady-state.

Values of $\delta_{p h}$ throughout the grid can easily be extracted from the FLUENT/SF code. This was done in three ways: firstly it was calculated from the plain advection-diffusion-reaction solution. Secondly it was calculated using the mean species and temperature values from the SF solution at each point, known here as 'defect of means.' Finally $\delta_{p h}$ was calculated for each field at each grid node and the mean and rms of this taken, known as 'mean defect' and 'rms defect.' The results here, Fig. 8, agree with those seen in Baker et al. (2004); Baik et al. (2007) with low positive values found inside the canyon 
and a ridge of high values along the mixing layer at the top of the canyon. This indicates that the chemistry is close to equilibrium in the canyon but is far from equilibrium at roof-top level where polluted air from the canyon is mixed with fresh, $\mathrm{O}_{3}$ rich, air from above. Also, as expected, a departure from equilibrium is observed around the source where polluted air is mixed into the canyon.

Fig. 8 shows $\delta_{p h}$ along a vertical line through the canyon. Also shown here is the defect as calculated using the plain method. We see that the SF defect of mean values are slightly higher than when fluctuations are ignored, suggesting that there are small differences in calculated values of means that are not apparent when considered individually. The peak true mean defect is significantly smaller and narrower than those using mean quantities, indicating that the chemistry is closer to equilibrium. The co-variance of $\mathrm{O}_{3}$ and $\mathrm{NO}$ caused by their opposite gradients in the mixing region will have an effect on mean $\delta_{p h}$ which, unlike reaction rate, will be unaffected by micromixing. This is further evidence to suggest that the composition found in the mixing layer at a given instant will retain some of it's 'in canyon' or 'out of canyon' equilibrium composition rather than being a fully mixed intermediate composition (which would be further from chemical equilibrium).

\section{$3.4 \quad C B M-I V$ Chemistry}

For the simulations with the CBM-IV chemistry $\mathrm{NO}, \mathrm{NO}_{2}$ and $\mathrm{O}_{3}$ levels for both background and source were kept the same as for the simple chemistry. Many of the species used in the CBM-IV mechanism are lumped species representing a number of actual species. A $V O C / N O_{x}$ ratio of 1.0 (by vol-

ume fraction) was used for the source, the VOC's comprised $70 \%$ Paraffin, 
$P A R$ and $5 \%$ each of Ethene (ETH), Olefinic carbon bond (OLE), Aldehydes $(A L D)$, Formaldehyde $(F O R M)$, Xylene $(X Y L)$ and Toluene $(T O L)$. In the background these VOC's were set to relative low levels compared to those at the source, $9.0 \mathrm{ppb}$ in total split in the same proportion as for the source. $C O$ was set to $1000 \mathrm{ppb}$ in the background and $40 \mathrm{ppb}$ at the source, $\mathrm{OH}$ and $\mathrm{HO}_{2}$ were set to $4 \times 10^{-6} \mathrm{ppb}$ and $4 \times 10^{-5} \mathrm{ppb}$ respectively for both source and background. All other species were set to small values and allowed to achieve steady-state levels during the calculation.

Contours for those species which appear in both chemistries used here were found to be same with the CBM-IV mechanism as with the simple chemistry within $\sim 1 \mathrm{ppb}$. This suggests that the use of the simple chemistry in the street canyon situation is valid for finding $\mathrm{NO} / \mathrm{NO}_{2} / \mathrm{O}_{3}$ levels. Again the levels of these species were found to be unaffected by segregation effects. There was also no significant difference observed for $V O C$ 's or secondary pollutants such as $\mathrm{HNO}_{2}$ and $\mathrm{HNO}_{3}$.

However significant differences were observed for some species between the plain advection-diffusion-reaction and Stochastic Fields results. These differences were observed in the mixing region above the top of the canyon where strong gradients and mixing occurs, as shown by the high variance in this region (see Fig. 5). The variation of mean and rms volume fraction with height along a vertical through the centre of the canyon for $\mathrm{OH}$ is shown in Fig. 9 to illustrate this. It can be seen that when segregation is ignored the peak $O H$ level observed in the mixing layer is too large and its vertical position is too high.

The effect of sample size on statistical accuracy can be considered using the central limit theorem (Tennekes and Lumley, 1972). This states that the stan- 
dard deviation of the statistical error in calculating mean quantities is given by:

$$
E_{s}=\frac{\sigma}{\sqrt{F}}
$$

where $\sigma$ is the standard deviation of the measured quantity and $F$ is the number of samples (or fields in this case). For the results produced using the CBM-IV chemistry and 14 fields radicals, such as $\mathrm{OH}$, in the mixing region at the top of the canyon show the highest rms values relative to the mean. The peak rms here is approximately $65 \%$ of the mean, using the central limit theorem this gives a relative statistical error of the mean of $17 \%$ (with 60 fields this error would be $8 \%$ ). This error is substantially smaller than the difference seen between the 'plain' and 'SF' results and hence it seems acceptable to draw conclusions from these results.

We again calculated $D a$ values for each species using the same method as in Section 3.2. As segregation effects were observed to have an effect in the mixing region the top of the canyon, a representative value of $D a$ for each species was obtained by taking an area-weighted average in a region covering the width of the canyon and extending from a height of $20 \mathrm{~m}$ to $35 \mathrm{~m}$, i.e. from $4 \mathrm{~m}$ below roof level to $11 \mathrm{~m}$ above. These values are given in Table 2 for the twelve 'fastest' species as calculated using this method. Vertical profiles of mean volume fraction though this region, along a line in the centre of the canyon, are shown in Fig. 10.

We see strong agreement between high $D a$ and a significant segregation effect on calculated species concentration. Those species for which large differences were seen between the two methods were the seven fastest species, namely $O H$, $\mathrm{NO}_{3}, \mathrm{C}_{2} \mathrm{O}_{3}, \mathrm{HO}_{2}, \mathrm{XO}_{2}$ (Peroxy radical), $\mathrm{XO}_{2} \mathrm{~N}$ (Peroxy radical accounting for additional nitrate) and PHO (higher molecular weight phenols). These 
species all had $D a$ values greater than approximately $\sim 1-20$. Those with lower $D a$, including those not shown in Fig. 10, did not show a significant segregation effect. This finding agrees with Garmory et al. (2008), where for species reacting in a jet exhaust plume it was found that those species affected by segregation were those with $D a$ greater than $\sim 5$. The Computational Singular Perturbation (CSP) method has been used to calculate chemical timescales for the CBM-IV mechanism (Neophytou et al., 2004). The seven fastest species found using the CSP method for their daytime, urban case were found to be the same seven species found here to show significant effects due to segregation. These results also agree with those for photostationary state defect which showed that the chemistry in the mixing layer is closer to equilibrium locally in the mixing layer than is suggested by use of only mean quantities. The peak in concentration of radicals such as $O H$ is caused by the departure from equilibrium at that point, a reduction of this peak indicates that the chemistry is closer to equilibrium.

\subsection{Conditional Statistics}

As with any PDF method, the Stochastic Fields method can provide any onepoint composition statistics. This includes conditional concentration statistics. By plotting a reactive scalar, such as $\mathrm{NO}_{2}$, from each field against an inert scalar from the same field it is possible to obtain scalar concentration statistics conditional on the value of a conserved scalar. This is shown for $\mathrm{NO}_{2}$ in Fig. 11. The results were obtained using the CBM-IV chemistry and results for all spatial nodes in the canyon up to a height of $20 \mathrm{~m}$ are plotted on the same figure. The conserved scalar used here is mixture fraction, $\xi$ which takes a value of zero in the background air and one at the source. This is found by

using a an extra inert scalar in each field in the simulation which takes a 
value of one at the source. Also shown for reference is the inert or 'frozen' relationship.

If $\mathrm{NO}_{2}$ did not undergo reaction it would vary linearly with mixture fraction between its source and background concentrations. We can see that the $\mathrm{NO}_{2}$ varies almost linearly between its background value at $\xi=0$ fraction and a maximum value at approximately $\xi=0.24$, and again almost linearly from the maximum to the source value at $\xi=1$. We also see the conditional variance from the spread of the points about this trend. Experimental data for conditional concentration statistics of $\mathrm{NO}_{2}$ in Brown and Bilger (1998) show trends with mixture fraction that are more curved than that seen in Fig. 11. This indicates that there is a lower scalar dissipation rate here; that is the effect of molecular diffusion is lower here than for Brown and Bilger's experiment. Since the scalar dissipation rate is usually modelled as being proportional to the variance of mixture fraction, the relatively good mixing inside most of the canyon results in low mixture fraction variance and hence scalar dissipation, whereas Brown and Bilger's experiment was conducted using a confined laboratory plume where the variance was higher.

\subsection{Discussion}

Using the CBM-IV mechanism it has been found that seven species show significant differences in predicted concentration when segregation is considered. These were found to be those species with Damköhler numbers above a threshold value between approximately 1 and 20, which agrees with simulations of a jet engine exhaust (Garmory et al., 2008). Values of Da for most of the major species would be increased if higher concentrations of the species they react with are used, as this would lead to higher reaction rates. In addition to this, 
changes in the configuration of the street canyon or the flow inside it would cause the turbulent timescale to change; leading to a different timescale for molecular mixing. Hence while for the conditions modelled here there are no segregation effects for the major species, it cannot be said that this will be the case in all conditions.

Calculations of the photostationary state defect using the simple chemistry have shown that air in the mixing layer above the canyon is closer to chemical equilibrium when the value in the individual fields are considered rather than only mean species concentrations. This is supported by the observation that the peak in $O H$ concentration is also reduced when fluctuating values are considered. Together with the results seen for the higher moments of the PDF of the scalars seen in this region this seems to suggest that the composition of the air in this region if sampled instantaneously would tend to be largely either polluted air from inside the canyon or clean air from outside, rather than a well-mixed combination of the two.

The ability to easily extract one-point composition statistics such as variance or conditional statistics is one of the advantages of using a PDF method. The Stochastic Fields method allows PDF simulations of practical simulations such as street canyons to be easily set up using existing Eulerian CFD techniques. While to get a high level of statistical accuracy will necessarily be computationally expensive, the results obtained can be used to compare against other, less computationally expensive, methods.

As discussed by Baker et al. (2004) and Baik et al. (2007) there is currently no experimental data for reactive pollutant concentration in a street canyon with which to validate predictions such as contained in this paper. Finding such data from field trials is not practicable due to the uncontrollable nature 
of the flow field in a real world setting. Wind tunnel experiments would need to made using a different source and background composition to that of the real flow such that the Damköhler number of the reactions in the model are the same as for the real flow. This may preclude more complex chemistries.

\section{Conclusions}

The Stochastic Fields method has been used to investigate the effect of segregation on the chemistry in a street canyon using a simple reversible $\mathrm{NO}_{x} / \mathrm{O}_{3}$ chemistry and also the CBM-IV mechanism. It has been found that both mechanisms used give very similar predictions for $\mathrm{NO}_{2}$ and $\mathrm{O}_{3}$ concentrations. For most of the species segregation does not have a significant effect using either the simple or more complex chemical mechanisms. However for seven species $\left(\mathrm{NO}_{3}, \mathrm{OH}, \mathrm{HO}_{2}, \mathrm{C}_{2} \mathrm{O}_{3}, \mathrm{XO}_{2}, \mathrm{XO}_{2} \mathrm{~N}\right.$ and $\left.\mathrm{PHO}\right)$ a difference was seen in the predicted concentration in the mixing region above the top of the canyon. These were the seven species with the fastest chemistry, as found by the method described in Section 3.2 and by a more sophisticated method Neophytou et al. (2004). All other species were found to have Damköhler numbers of less than $\sim 5$.

The rms of concentration was found to be very high in the mixing layer at the top of the canyon, with fluctuations of the order of the mean values. Investigation of the higher moments of the PDF revealed that there is very low value of kurtosis in this region, indicating that a sample of air taken instantaneously at this point has a high likelihood of having a composition close to that 'in canyon' or 'out of canyon' rather than a well mixed combination of the two. This is supported by predictions for the photostationary state defect. Predictions found using the Stochastic Fields method gave lower values in the 
mixing region than those from a simulation using only mean concentrations, indicating that the chemistry is closer to equilibrium, as found above or below the mixing region, than a fully mixed mean concentration would suggest.

\section{Acknowledgements}

This work has been funded by the Engineering and Physical Sciences Research Council.

\section{References}

J-J. Baik and J-J. Kim. On the escape of pollutants from urban street canyons. Atmospheric Environment, 36:527-536, 2002.

J-J. Baik, Y-S. Kang, and J-J. Kim. Modelling reactive pollutant dispersion in an urban street canyon. Atmospheric Environment, 41:934-949, 2007.

J. Baker, H. L. Walker, and X. Cai. A study of the dispersion and transport of reactive pollutants in and above street canyons - a Large Eddy Simulation. Atmospheric Environment, 38:6883-6892, 2004.

R. J. Brown and R. W. Bilger. Experiments on a reacting plume - 2. conditional concentration statistics. Atmospheric Environment, 32:629-646, 1998.

G. D. Byrne. Pragmatic experiments with Krylov methods in the stiff ODE setting. In J. Cash and I. Gladwell, editors, Computational Ordinary Differential Equations. Oxford University Press, 1992.

L. J. Carpenter, K. C. Clemitshaw, R. A. Burgess, S. A. Penkett, J. N. Cape, and G. G. McFadyen. Investigation and evaluation of the $\mathrm{NO}_{x} / \mathrm{O}_{3}$ photochemical steady state. Atmospheric Environment, 32:3353-3365, 1998. 
M. Cassiani, P. Franzese, and U. Giostra. A PDF micromixing model of dispersion for atmospheric flow. Part I: development of the model, application to homogeneous turbulence and to neutral boundary layer. Atmospheric Environment, 39:1457-1469, 2005.

F. Caton, R. E. Britter, and S. Dalziel. Dispersion mechanisms in a street canyon. Atmospheric Environment, 37:693-702, 2003.

T. L. Chan, G. Dong, C. S. Cheung, C. W. Leung, C. P. Wong, and W. T. Wung. A Monte Carlo simulation of nitrogen oxides dispersion from a vehicular exhaust plume and its sensitivity studies. Atmospheric Environment, 35:6117-6127, 2001.

F. Costabile and I. Allegrini. Measurements and analyses of nitrogen oxides and ozone in the yard and on the roof of a street-canyon in Suzhou. Atmospheric Environment, 41:6637-6647, 2007.

N. S. Dixon and A. S. Tomlin. A Lagrangian stochastic model for predicting concentration fluctuations in urban areas. Atmospheric Environment, 41: 8114-8127, 2007.

R. O. Fox. Computational models for turbulent reacting flows. Cambridge University Press, Cambridge, 2003.

C. W. Gardiner. Handbook of stochastic methods. Springer, Berlin, third edition, 2004.

A. Garmory. Micromixing effects in atmospheric reacting flows. PhD Thesis, University of Cambridge, 2007.

A. Garmory, E. S. Richardson, and E. Mastorakos. Micromixing effects in a reacting flow by the Stochastic Fields method. Atmospheric Environment, 40:1078-1091, 2006.

A. Garmory, R. E. Britter, and E. Mastorakos. Simulation of the evolution of aircraft exhaust plumes including detailed chemistry and segregation. Journal of Geophysical Research, 113:D08303, 2008. 
M. Gery, G. Witten, J. Killus, and M. Dodge. A photochemical kinetics mechanism for urban and regional scale computer modelling. Journal of Geophysical Research, 94(D10):12925-12956, 1989.

D. Hamlyn and R. E. Britter. A numerical study of the flow field and exchange processes within a canopy of urban-type roughness. Atmospheric Environment, 39:3243-3254, 2005.

W. G. Hoydysh and W. F. Dabberdt. Kinematics and dispersion characteristics of flows in asymmetric street canyons. Atmospheric Environment, 22: 2677-2689, 1988.

S. J. Jeong and M. J. Andrews. Application of the $k-\epsilon$ turbulence model to the high Reynolds number skimming flow field of an urban street canyon. Atmospheric Environment, 36:1137-1145, 2002.

C-H. Lui and M. C. Barth. Large-eddy simulation of flow and scalar transport in a modelled street canyon. Journal of Applied Meteorology, 41:660-673, 2002.

R. Mustata, L. Valiño, C. Jiménez, W. P. Jones, and S. Bondi. A Probability Density Function Eulerian Monte Carlo field method for Large-eddy simulations. Application to a turbulent piloted methane/air diffusion flame (Sandia D). Combustion and Flame, 145:88-104, 2006.

M. Neophytou, D. A. Goussis, M. van Loon, and E. Mastorakos. Reduced chemical mechanisms for atmospheric pollution using Computational Singular Perturbation analysis. Atmospheric Environment, 38:3661-3673, 2004.

M. Pavageau and M. Schatzmann. Wind tunnel measurements of concentration fluctuations in an urban street canyon. Atmospheric Environment, 33: 3961-3971, 1999.

S. B. Pope. Lagrangian PDF methods for turbulent flows. Annual Review of Fluid Mechanics, 26:23-63, 1994.

M. W. Rotach. Profiles of turbulent statistics in and above an urban street 
canyon. Atmospheric Environment, 29:1473-1486, 1995.

V. Sabel'nikov and O. Soulard. Simulation of a turbulent premixed methane flame with an Eulerian Monte Carlo solver. In V. Dias and J. Vandooren, editors, Proceedings of the European Combustion Meeting, page E100, Louvain, 2005a. Belgian Section of The Combustion Institute.

V. Sabel'nikov and O. Soulard. Rapidly decorrelating velocity-field model as a tool for solving one-point Fokker-Planck equations for probability density functions of turbulent reactive scalars. Physical Review E, 72(1):Article No. 016301, 2005b.

O. Soulard and V. Sabel'nikov. Eulerian Monte Carlo method for the joint velocity and mass-fraction probability density function in turbulent reactive gas flows. Combustion, Explosion and Shock Waves, 42:753-762, 2006.

H. Tennekes and J. L. Lumley. A first course in turbulence. MIT Press, Massachusetts, 1972.

L. Valiño. A field Monte Carlo formulation for calculating the probability density function of a single scalar in a turbulent flow. Flow Turbulence and Combustion, 60:157-172, 1998.

A. Walton and A. Y. S. Cheng. Large-eddy simulation of pollution dispersion in an urban street canyon - Part II: idealised canyon simulation. Atmospheric Environment, 36:3615-3627, 2002. 
Fig. 1. Schematic diagram of 2D street canyon.

Fig. 2. Mean velocity vectors from CFD solution.

Fig. 3. Contours of Mixture Fraction in and above canyon. (a) Mean values; (b) rms.

Fig. 4. Profiles of concentration of inert scalar on leeward and windward faces, normalised by concentration at ground level on leeward wall. Experimental data from Hoydysh and Dabberdt (1988).

Fig. 5. Contours (in ppb) of $\mathrm{NO}_{2}$ distribution in and above canyon. (a) Mean values; (b) rms; (c) skewness (Eq. (3)) and (d) kurtosis excess (Eq. (4)) with positive values omitted.

Fig. 6. Probability Density Function of $\mathrm{NO}_{2}$ volume fraction at roof-top height $(24 \mathrm{~m})$ in the centre of the canyon. Also indicated are the background concentration, $0.2 \mathrm{ppb}$, and the source concentration, $10 \mathrm{ppb}$.

Fig. 7. Profiles of mean and rms $O_{3}$ concentrations, using both SF and plain advection-diffusion-reaction model, taken along a vertical line extending through the centre of the canyon passing through the source 
Fig. 8. Vertical profiles of photostationary state defect through the centre of the street

Fig. 9. Vertical profiles of $O H 2.5 \mathrm{~m}$ through the centre of the street, using CBM-IV chemistry.

Fig. 10. Vertical profiles, between a height of $20 \mathrm{~m}$ and $35 \mathrm{~m}$ on the canyon centre-line, of volume fraction for the twelve species with the highest $D a$ at the centre of the canyon, using CBM-IV chemistry. Key to labels given in Table 2.

Fig. 11. Conditional dependence of $\mathrm{NO}_{2}$ volume fraction with mixture fraction as produced by the SF method using the CBM-IV chemistry. Also shown is the dependence if no reaction were to take place. 
Table 1

Solution parameters used in FLUENT CFD solution

\begin{tabular}{|l|l|}
\hline Solver & $2 \mathrm{D}$, segregated, implicit \\
Pressure-velocity coupling & SIMPLE \\
Density & Constant \\
Discretisation - pressure & Standard \\
Discretisation - momentum & $1^{\text {st }}$-order upwind \\
Discretisation - $\mathrm{k}$ & $1^{\text {st }}$-order upwind \\
Discretisation - $\epsilon$ & $1^{\text {st }}$-order upwind \\
\hline
\end{tabular}


Table 1

Area-weighted averages of Damköhler numbers in the mixing region for the twelve fastest species using the CBM-IV mechanism. Also shown is the key to the corresponding figure in Fig. 8

\begin{tabular}{|l|r|r|}
\hline Species & $D a$ & Position in Fig. 8 \\
\hline $\mathrm{OH}$ & 389 & $(\mathrm{a})$ \\
$\mathrm{NO}_{3}$ & 387 & $(\mathrm{~b})$ \\
$\mathrm{C}_{2} \mathrm{O}_{3}$ & 199 & $(\mathrm{c})$ \\
$\mathrm{HO} \mathrm{O}_{2}$ & 120 & $(\mathrm{~d})$ \\
$\mathrm{XO} \mathrm{O}_{2} \mathrm{~N}$ & 111 & $(\mathrm{e})$ \\
$\mathrm{XO}$ & 111 & $(\mathrm{f})$ \\
$\mathrm{PHO}$ & 20.0 & $(\mathrm{~g})$ \\
$\mathrm{PH} \mathrm{N}_{2} \mathrm{O}_{5}$ & 0.469 & $(\mathrm{l} N$ \\
$\mathrm{NO}$ & 0.00158 & $(\mathrm{l})$ \\
\hline
\end{tabular}



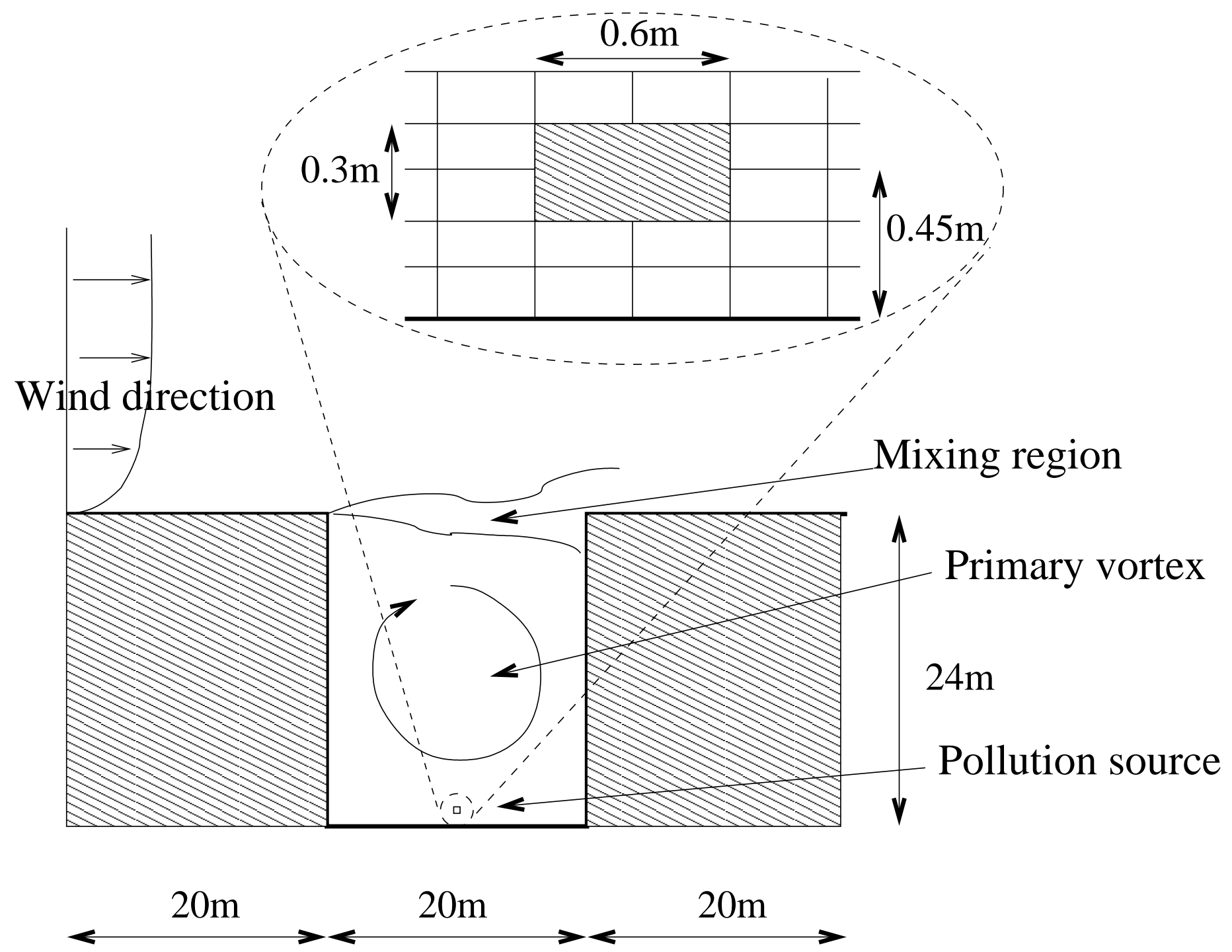
Figure 2

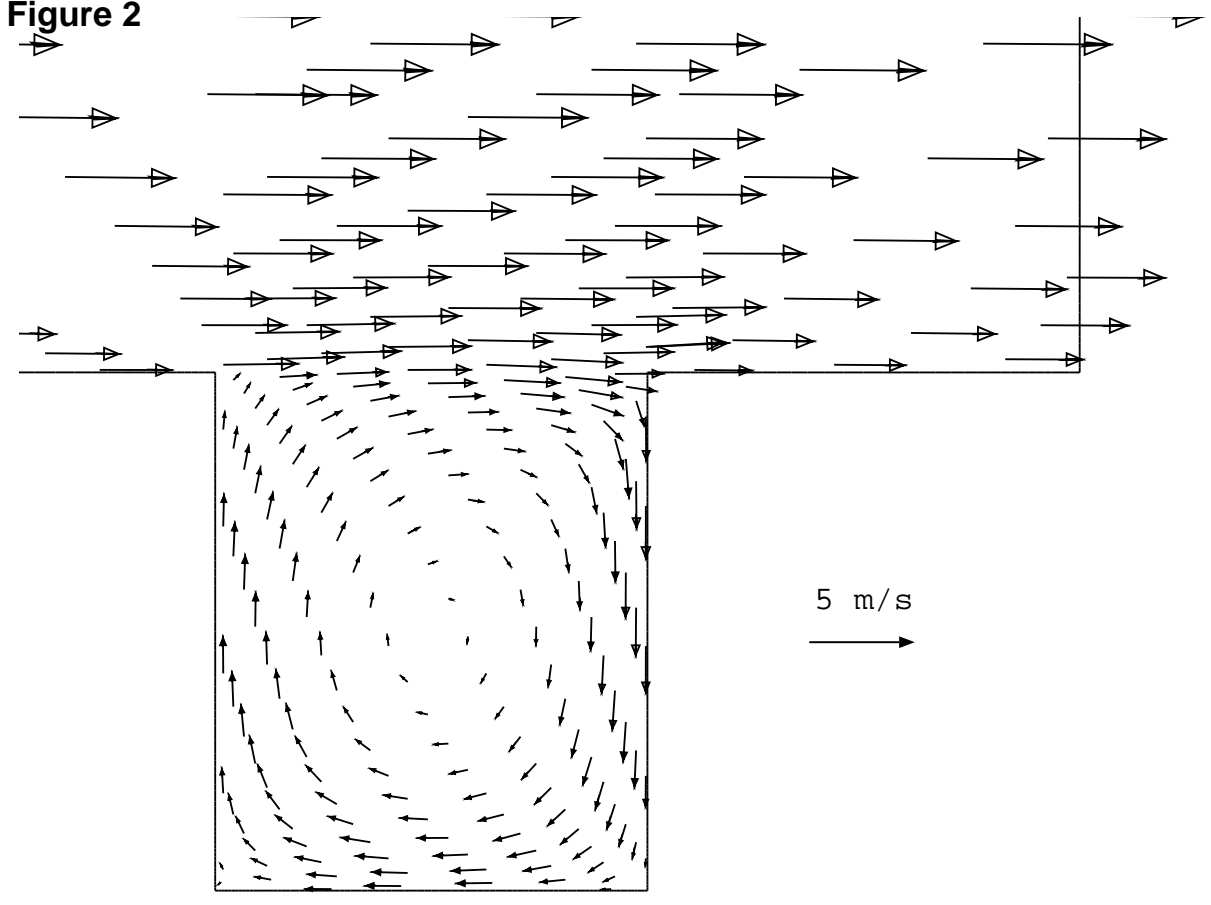


Figure 4

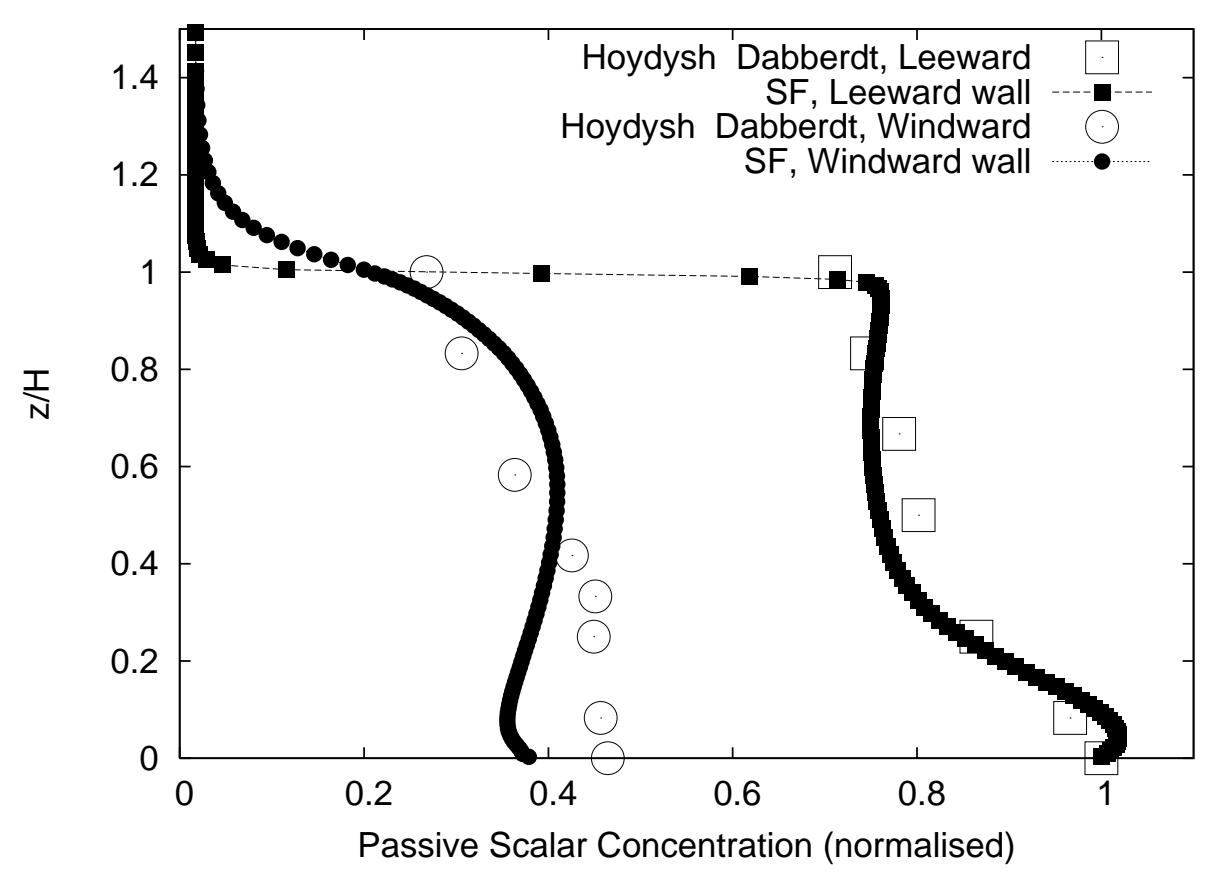
(1) 
Figure 5

$2.31 e+01$

$2.08 \mathrm{e}+01$

$1.85 \mathrm{e}+01$

$1.62 \mathrm{e}+01$

$1.39 \mathrm{e}+01$

$1.17 e+01$

$9.36 e+00$

$7.07 e+00$

$4.78 e+00$

$2.48 \mathrm{e}+00$

1.92e-01

(a)

$5.53 e+00$
$4.51 e+00$
$3.50 e+00$
$2.48 e+00$
$1.47 e+00$
$4.50 e-01$
$-5.65 e-01$
$-1.58 e+00$
$-2.60 e+00$
$-3.61 e+00$
$-4.63 e+00$

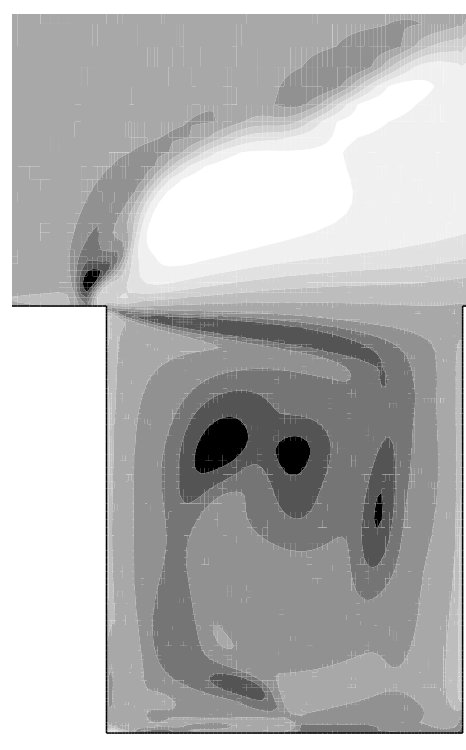

(c)
$6.61 e+00$

$5.95 e+00$

$5.29 e+00$

$4.63 e+00$

$3.97 e+00$

$3.31 e+00$

$2.65 e+00$

$1.98 \mathrm{e}+00$

$1.32 \mathrm{e}+00$

6.61e-01

$4.68 \mathrm{e}-05$

(b)

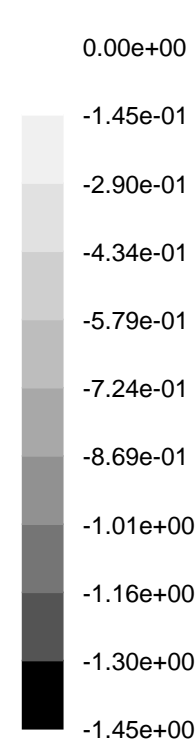

$-1.45 e+00$

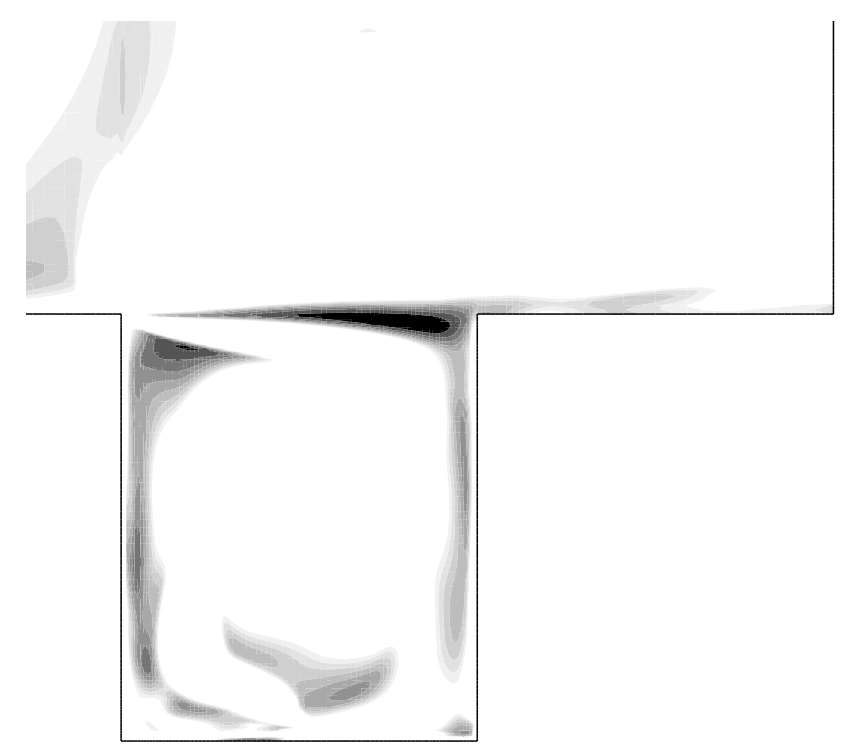

(d) 
Figure 6

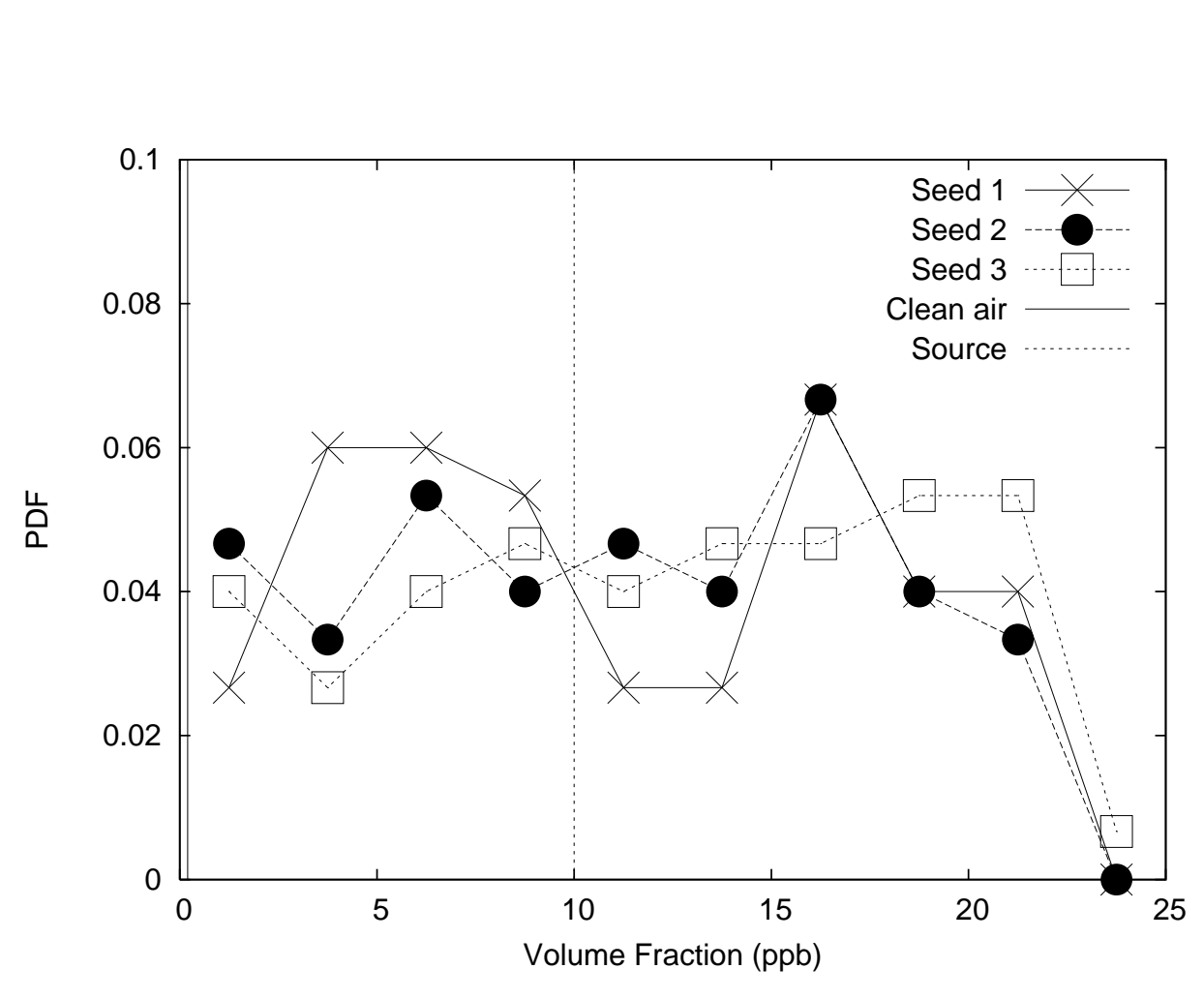

\section{Figure 6}

gure 6

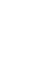
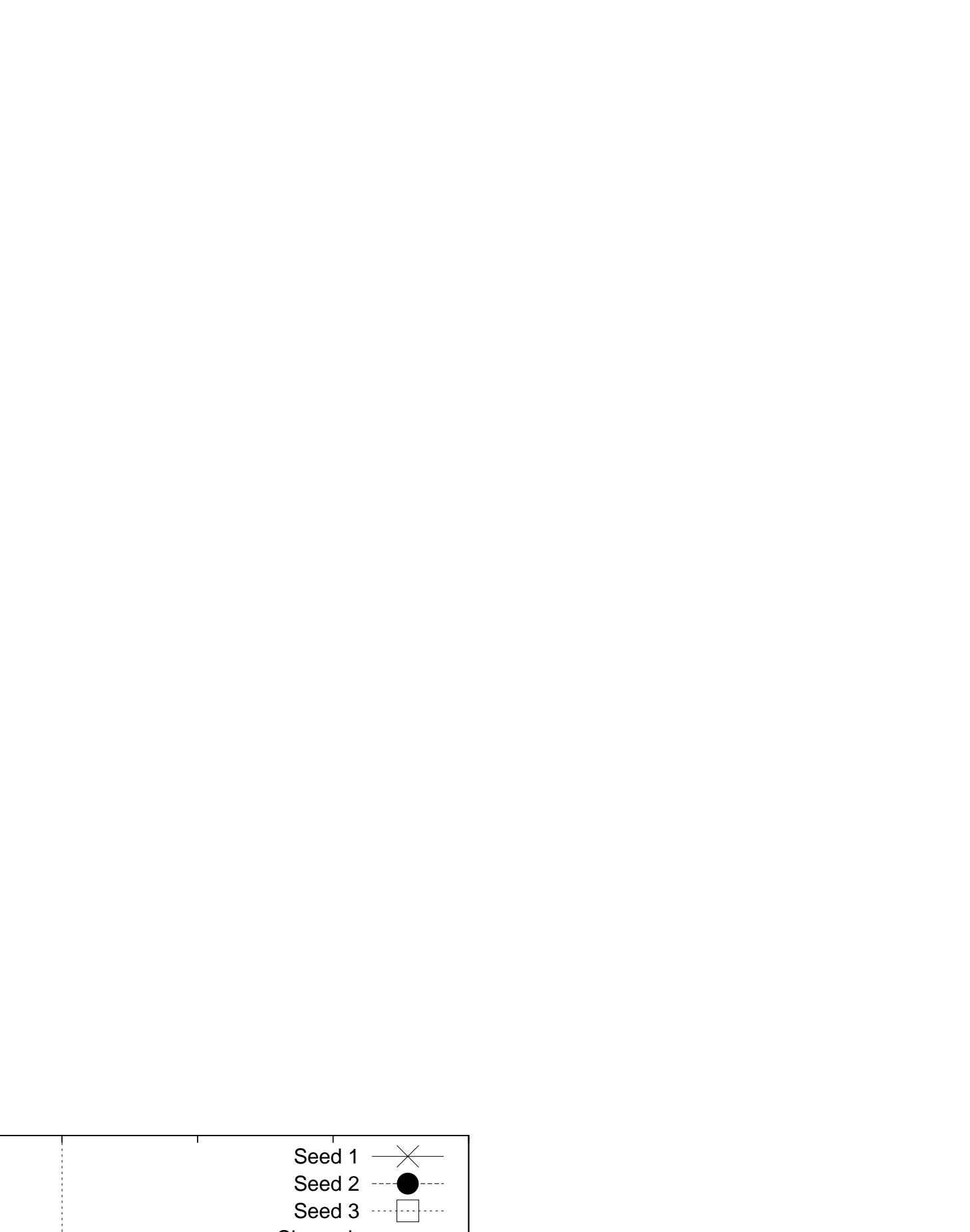
Figure 7

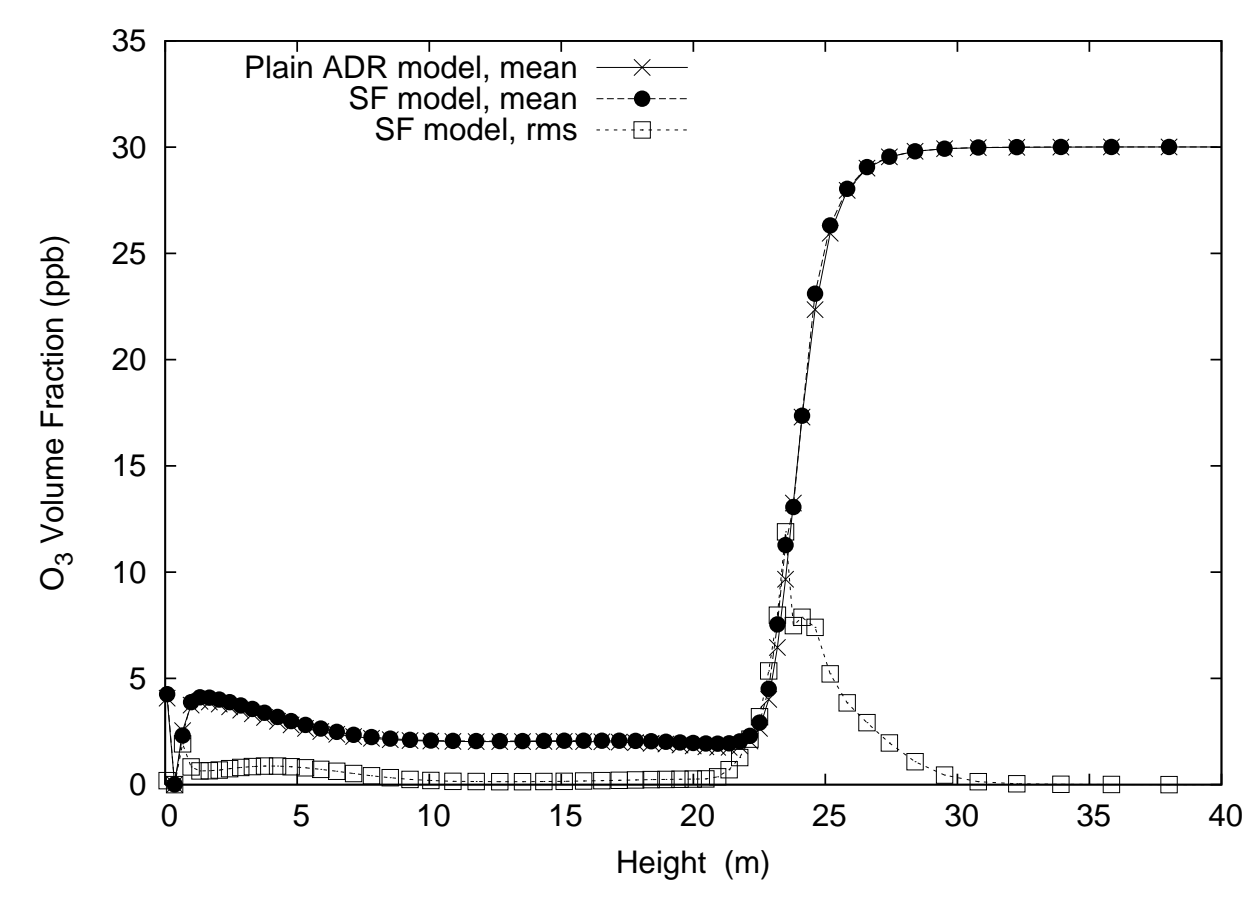

Figure 7

,

(n)

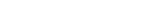

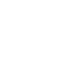

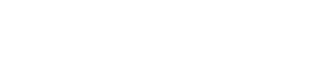

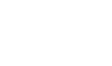

-

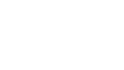

-

(2)

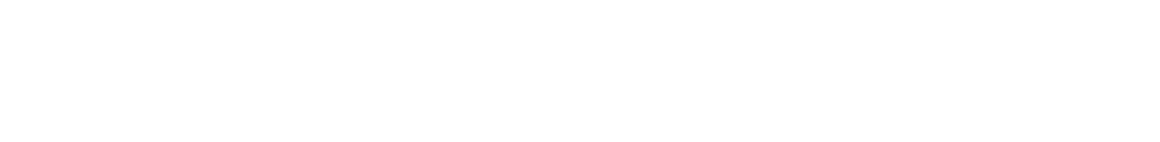

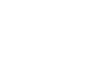
(2)

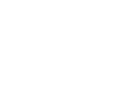

Height (m)

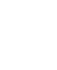

(1)

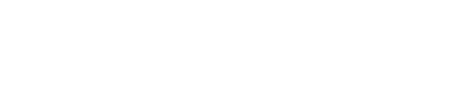




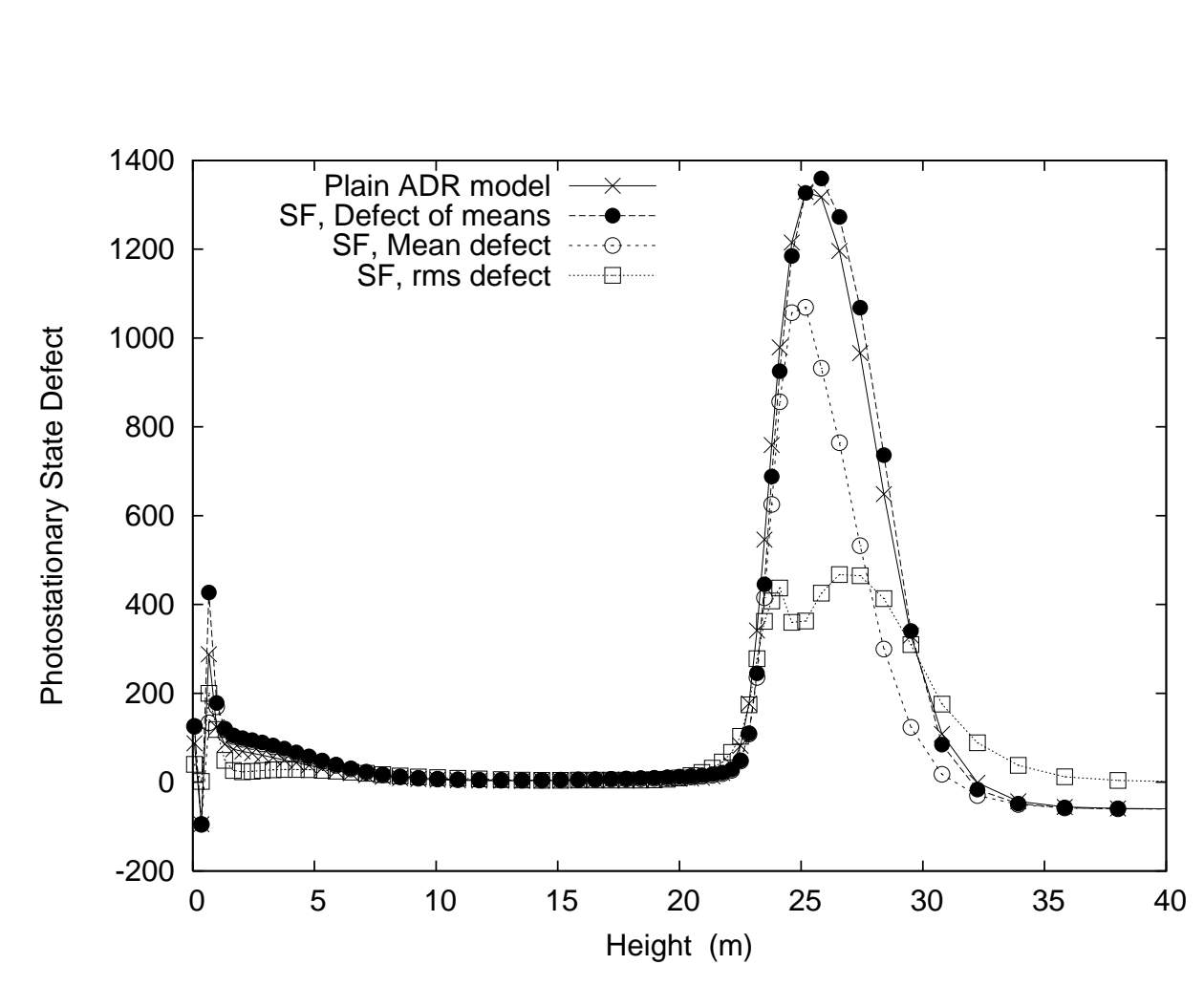

Figure 8

\section{Figure 8}

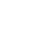
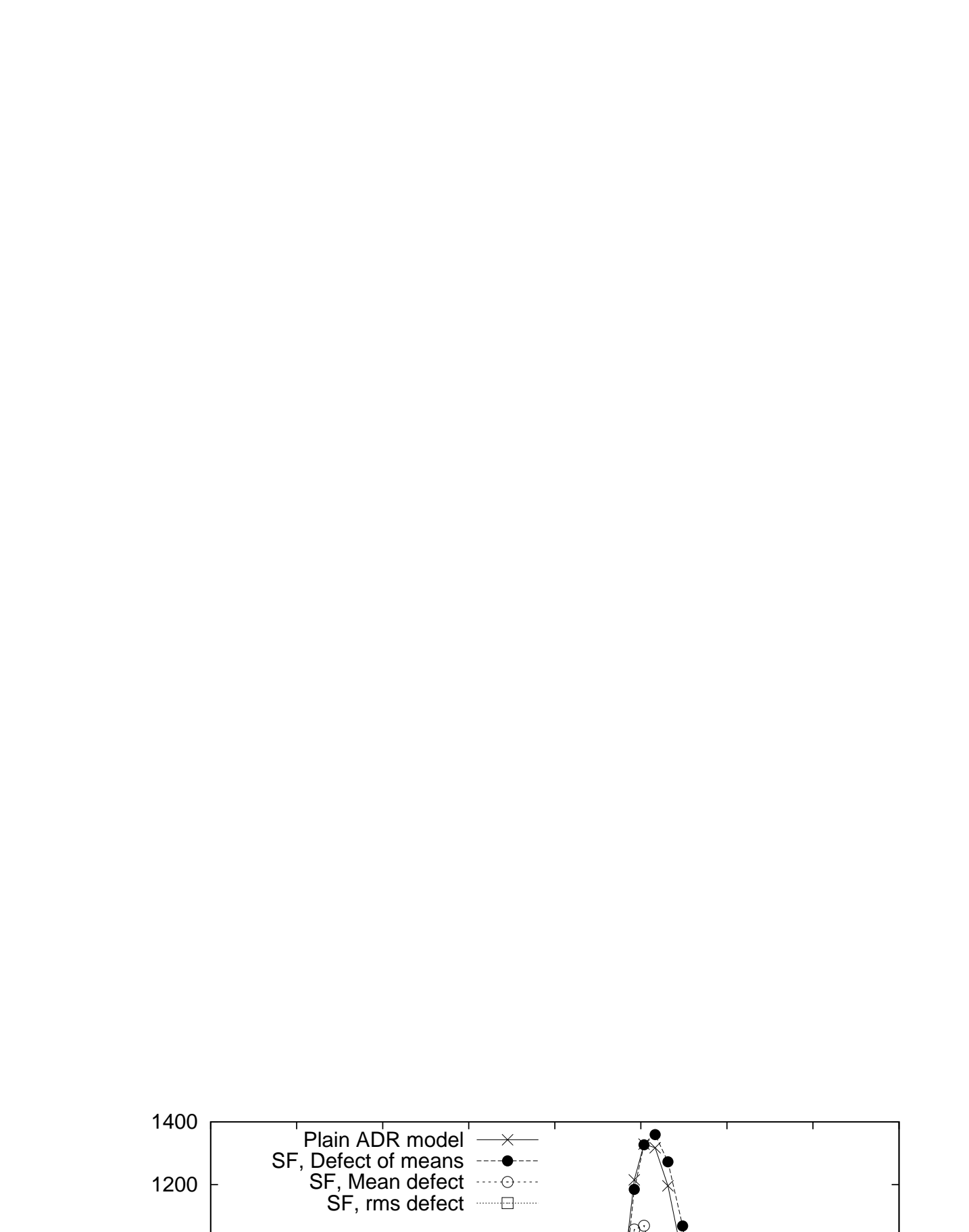

(2)

(2)

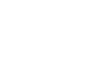

-

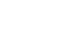

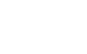

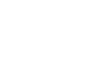

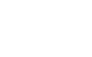

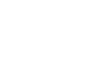
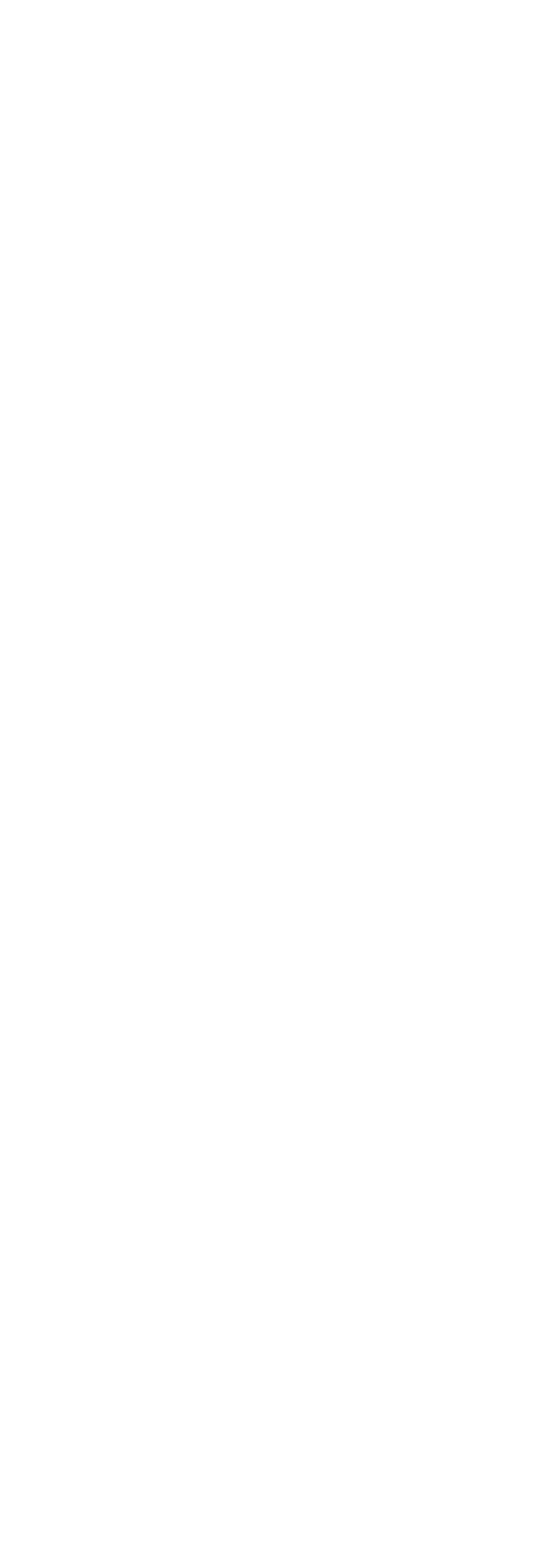
Figure 9

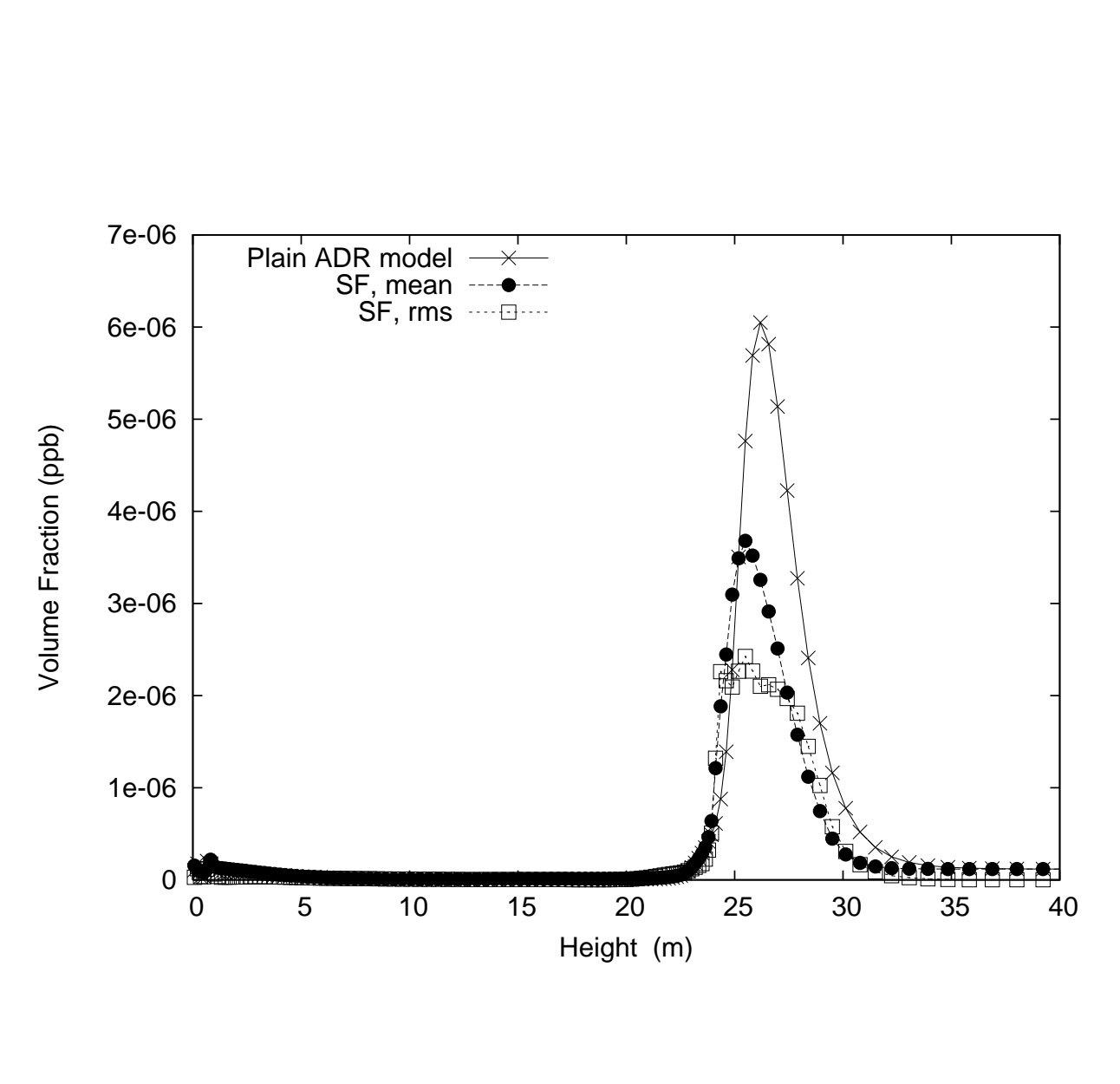

inde

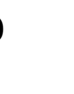

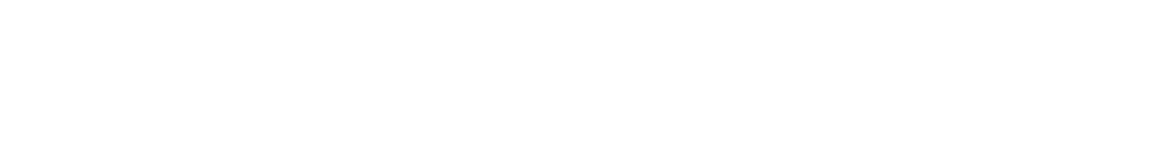

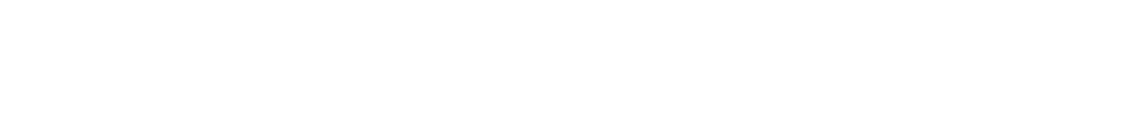

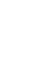


Figure 10 10lain -

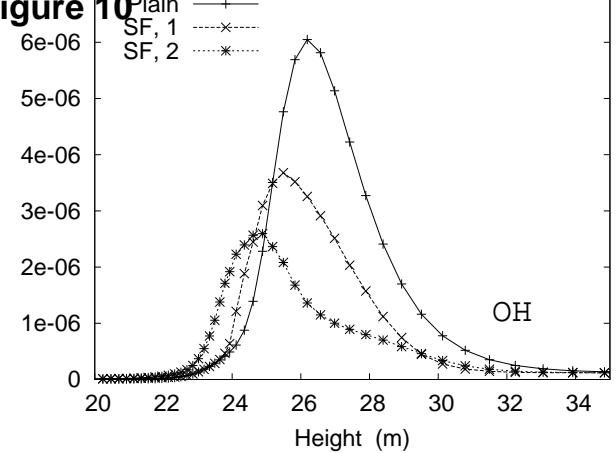

(a)

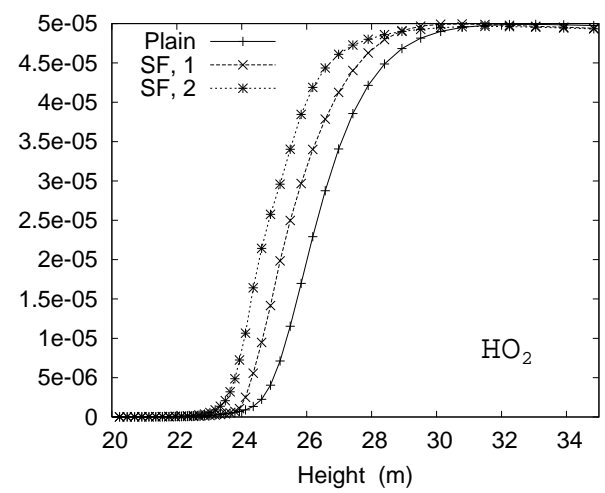

(d)

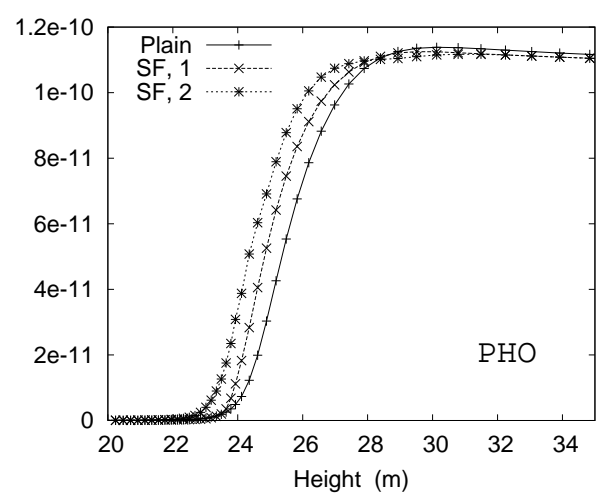

(g)

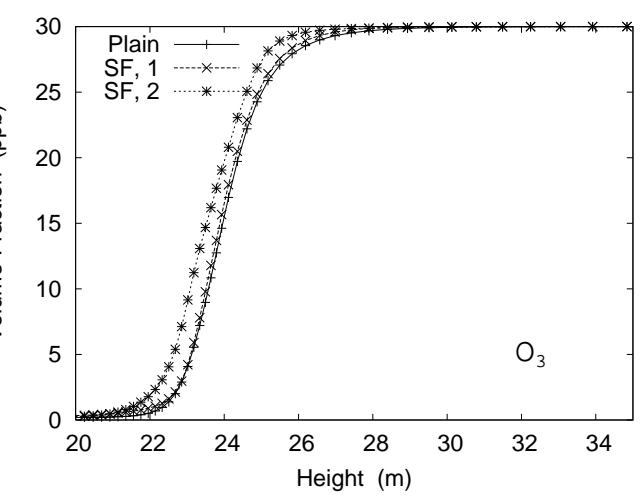

(j)

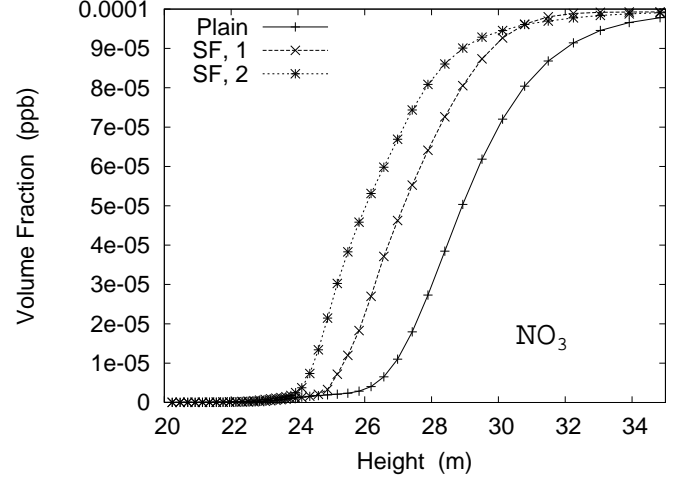

(b)

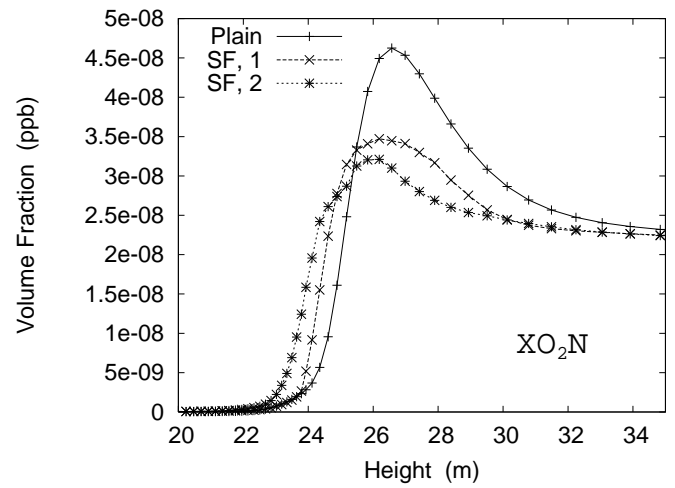

(e)

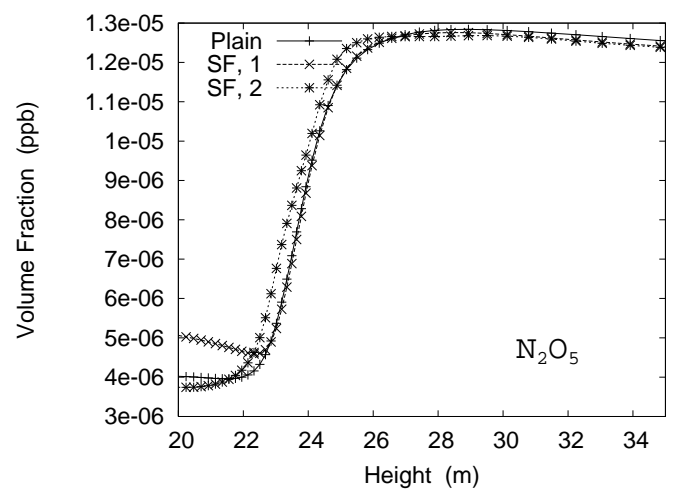

(h)

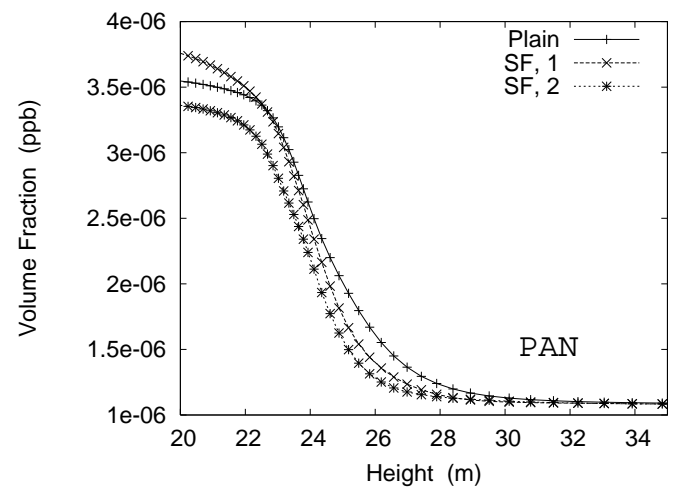

(k)

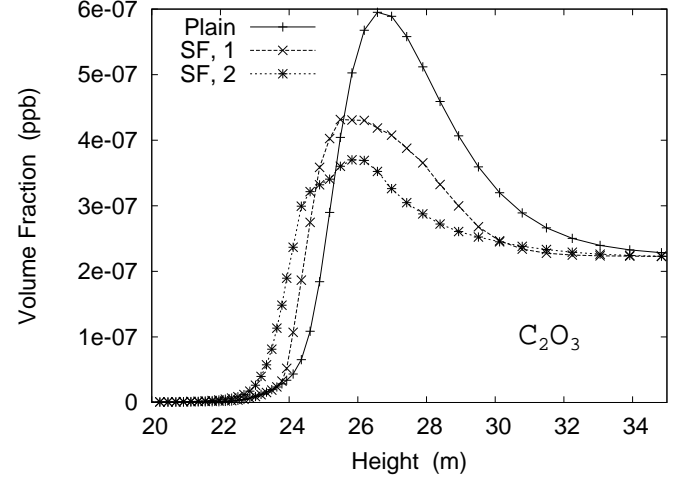

(c)

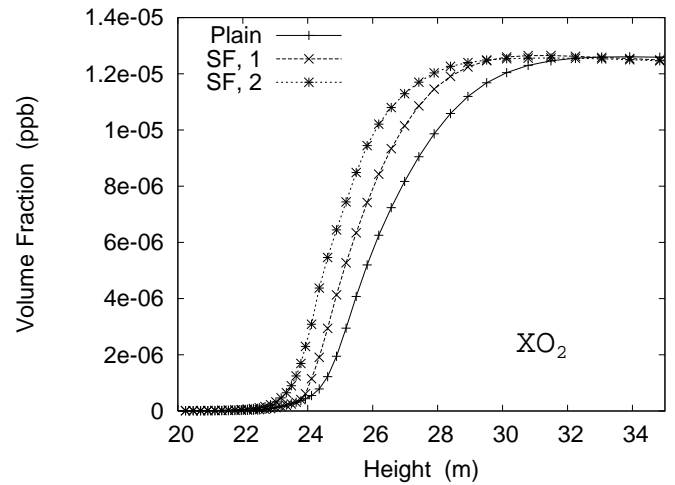

(f)

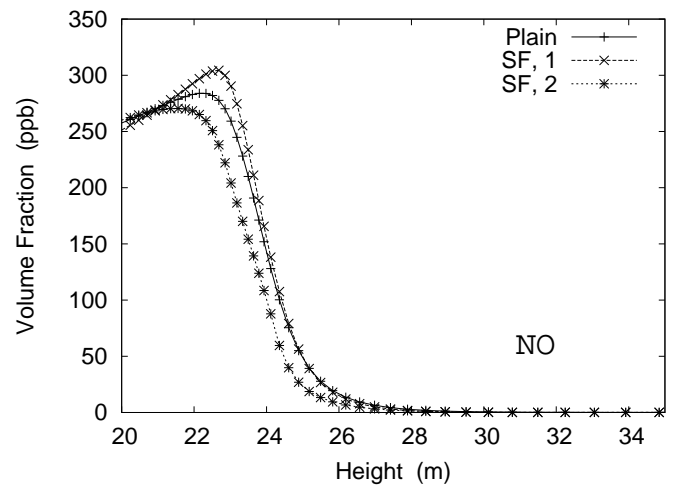

(i)

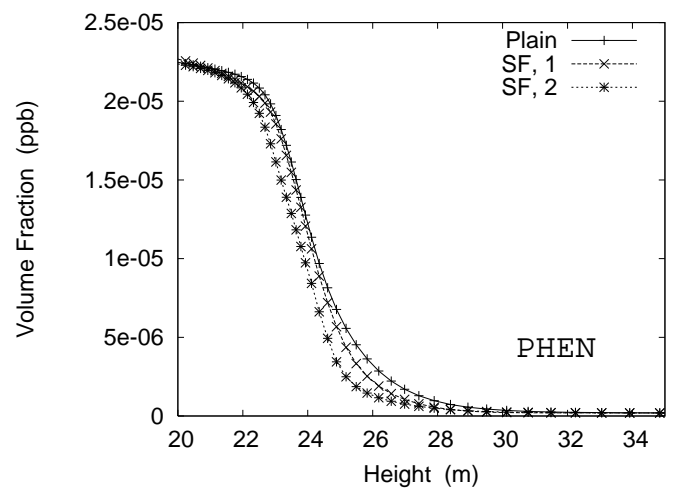

(1) 


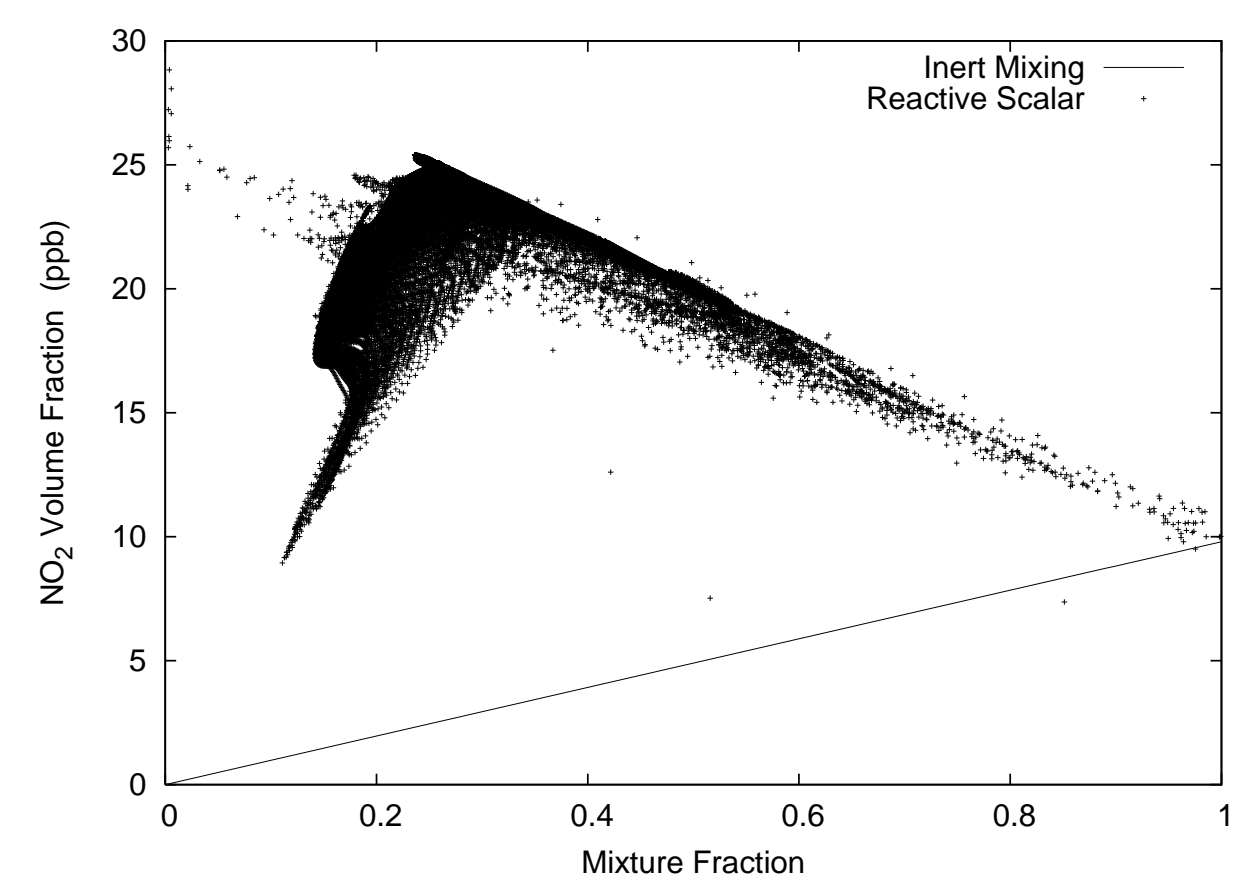

Figure 11

\author{
Mixture Fraction
}
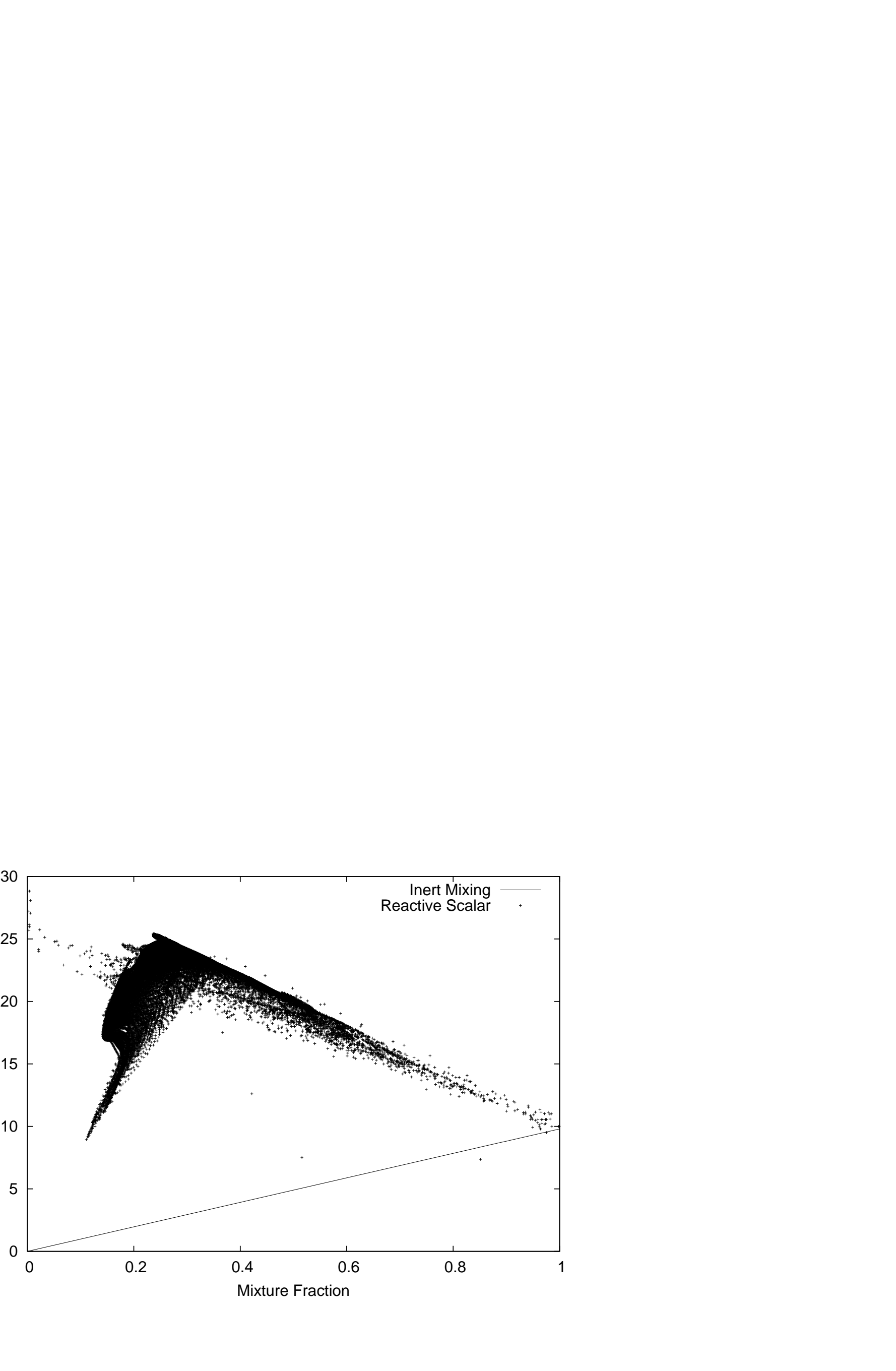